\title{
Nodeless superconductivity and preserved time-reversal symmetry in the noncentrosymmetric $\mathrm{Mo}_{3} \mathrm{P}$ superconductor
}

\author{
T. Shang,,${ }^{1,}$ J. Philippe, ${ }^{2}$ J. A. T. Verezhak,${ }^{3}$ Z. Guguchia, ${ }^{3}$ J. Z. Zhao, ${ }^{4}$ L.-J. Chang,,${ }^{5}$ M. K. Lee, ${ }^{5}$ D. J. Gawryluk,,${ }^{1,}$ \\ E. Pomjakushina, ${ }^{1}$ M. Shi, ${ }^{6}$ M. Medarde,${ }^{1}$ H.-R. Ott,${ }^{2,7}$ and T. Shiroka ${ }^{2,7}$ \\ ${ }^{1}$ Laboratory for Multiscale Materials Experiments, Paul Scherrer Institut, Villigen CH-5232, Switzerland \\ ${ }^{2}$ Laboratorium für Festkörperphysik, ETH Zürich, CH-8093 Zurich, Switzerland \\ ${ }^{3}$ Laboratory for Muon-Spin Spectroscopy, Paul Scherrer Institut, CH-5232 Villigen PSI, Switzerland \\ ${ }^{4}$ Co-Innovation Center for New Energetic Materials, Southwest University of Science and Technology, \\ Mianyang 621010, People's Republic of China \\ ${ }^{5}$ Department of Physics, National Cheng Kung University, Tainan 70101, Taiwan \\ ${ }^{6}$ Swiss Light Source, Paul Scherrer Institut, Villigen $\mathrm{CH}-5232$, Switzerland \\ ${ }^{7}$ Paul Scherrer Institut, CH-5232 Villigen PSI, Switzerland
}

(Received 8 March 2019; revised manuscript received 9 May 2019; published 23 May 2019)

\begin{abstract}
We report a comprehensive study of the noncentrosymmetric superconductor $\mathrm{Mo}_{3} \mathrm{P}$. Its bulk superconductivity, with $T_{c}=5.5 \mathrm{~K}$, was characterized via electrical-resistivity, magnetization, and heat-capacity measurements, while its microscopic electronic properties were investigated by means of muon-spin rotation/relaxation $(\mu \mathrm{SR})$ and nuclear magnetic resonance (NMR) techniques. In the normal state, NMR relaxation data indicate an almost ideal metallic behavior, confirmed by band-structure calculations, which suggest a relatively high electron density of states, dominated by the Mo $4 d$ orbitals. The low-temperature superfluid density, determined via transverse-field $\mu \mathrm{SR}$ and electronic specific heat, suggest a fully gapped superconducting state in $\mathrm{Mo}_{3} \mathrm{P}$, with zero-temperature gap $\Delta_{0}=0.83 \mathrm{meV}$, the same as the BCS gap value in the weak-coupling case, and a zero-temperature magnetic penetration depth $\lambda_{0}=126 \mathrm{~nm}$. The absence of spontaneous magnetic fields below the onset of superconductivity, as determined from zero-field $\mu$ SR measurements, indicates a preserved time-reversal symmetry in the superconducting state of $\mathrm{Mo}_{3} \mathrm{P}$ and, hence, spin-singlet pairing.
\end{abstract}

DOI: 10.1103/PhysRevB.99.184513

\section{INTRODUCTION}

Noncentrosymmetric superconductors (NCSCs) belong to a class of materials that miss a key symmetry, such as parity [1]. In NCSCs the lack of inversion symmetry of the crystal lattice often induces an antisymmetric spin-orbit coupling (ASOC), which lifts the degeneracy of the conduction-band electrons and splits the Fermi surface. Consequently, both intra- and interband Cooper pairs can be formed. The admixture of spin-singlet and spin-triplet pairing in NCSCs is determined by the strength of the ASOC and by other microscopic parameters [1,2]. Cooper pairs with a spin-triplet pairing have a nonzero spin, which implies a weak magnetic moment in the superconducting state and a breaking of the time-reversal symmetry (TRS). This is the case of, e.g., $\mathrm{Sr}_{2} \mathrm{RuO}_{4}$ and $\mathrm{UPt}_{3}$, known to exhibit triplet superconductivity [3-6], and where the TRS breaking in the SC state has been confirmed by measurements of both zero-field muon-spin relaxation/rotation $(\mu \mathrm{SR})$ and the polar Kerr effect [7-10].

Some NCSCs are known to show a broken TRS upon the onset of superconductivity. Examples include $\mathrm{LaNiC}_{2}$ [11],

\footnotetext{
*Corresponding author: tian.shang@psi.ch

$\dagger$ On leave from Institute of Physics, Polish Academy of Sciences, Aleja Lotnikow 32/46, PL-02-668 Warsaw, Poland.
}

$\mathrm{La}_{7} \operatorname{Ir}_{3}$ [12], and some Re-based binary alloys $\operatorname{Re} T(T=$ transition metal, e.g., Ti, $\mathrm{Zr}, \mathrm{Nb}, \mathrm{Hf}$ ) [13-16]. In the weakSOC limit, TRS breaking can be achieved via nonunitary triplet pairing, such as in noncentrosymmetric $\mathrm{LaNiC}_{2}[11,17]$ and centrosymmetric $\mathrm{LaNiGa}_{2}[18,19]$. In the case of $\mathrm{Re} T$ alloys, where a strong SOC is present, the $T_{d}$ point group has several irreducible representations with dimension larger than 1 . Therefore, they can support TRS breaking with singlet, triplet, or admixed pairings. For $\operatorname{Re} T$, there are a number of possible TRS-breaking states [16]; however, all such states have symmetry-constrained point or line nodes in the gap, inconsistent with the experimentally observed nodeless gap [13-16]. To explain a fully gapped superconducting state exhibiting broken TRS, a model employing loop Josephson currents (LJCs) was recently proposed [20]. This model is based on an on-site, intraorbital, singlet-pairing SC state, where a phase shift among the on-site singlet pairs gives rise to the LJCs within a unit cell. Such currents can produce weak internal magnetic fields, which break the TRS and can, in principle, be detected experimentally.

Despite numerous examples of NCSCs, to date only a few of them are known to break TRS in their superconducting state. The causes of such a selective TRS breaking remain largely unknown. Consequently, comprehensive studies of other NCSCs, such as $\mathrm{Mo}_{3} \mathrm{P}$ reported here, may help to identify the origin of this behavior. Superconductivity in 
$\mathrm{Mo}_{3} \mathrm{P}$ was first reported in 1954 [21] and confirmed ten years later [22]. In that same year, also the $\mathrm{Mo}_{3} \mathrm{P}$ crystal structure was determined [23]. To our knowledge, none of these early works had a follow-up regarding the characterization of the superconducting properties of $\mathrm{Mo}_{3} \mathrm{P}$.

In this paper, we report on an extensive study of the $\mathrm{Mo}_{3} \mathrm{P}$ physical properties, in both the normal and superconducting state, by means of electrical-resistivity, magnetization, thermodynamic, muon-spin relaxation $(\mu \mathrm{SR})$, and nuclear magnetic resonance (NMR) methods. In addition, we also present theoretical density functional theory (DFT) bandstructure calculations. Despite a noncentrosymmetric crystal structure, $\mathrm{Mo}_{3} \mathrm{P}$ is shown to be a moderately correlated electron material, which adopts a fully gapped, spin-singlet superconducting state with preserved TRS.

\section{EXPERIMENTAL DETAILS}

Polycrystalline samples of $\mathrm{Mo}_{3} \mathrm{P}$ were prepared via solidstate reaction, where high-purity (99.999\%) Mo powders and $\mathrm{P}$ pieces were mixed in a stoichiometric ratio and made to react at $1100^{\circ} \mathrm{C}$ for 48 hours. The resulting powders were thoroughly grounded and pressed into pellets and then sintered at $1100^{\circ} \mathrm{C}$ for over 48 hours. The final product had a silvery color, indicative of good metallicity. Room-temperature x-ray powder diffraction (XRD) measurements were performed by using a Bruker $\mathrm{D} 8$ diffractometer with $\mathrm{Cu} \mathrm{K} \alpha$ radiation. The magnetic-susceptibility, electrical resistivity, and specific-heat measurements were performed on a $7 \mathrm{~T}$ Quantum Design magnetic property measurement system (MPMS-7) and a $14 \mathrm{~T}$ physical property measurement system (PPMS-14) equipped with a dilution refrigerator (DR). The $\mu \mathrm{SR}$ measurements were carried out at the GPS and HAL-9500 spectrometers of the Swiss muon source at Paul Scherrer Institut, Villigen, Switzerland [24]. For the low-temperature HAL measurements, the samples were mounted on a silver plate using diluted GE varnish. The $\mu$ SR data were analyzed by means of the MUSRFIT software package [25].

The ${ }^{31} \mathrm{P}$ NMR measurements, including line shapes and spin-lattice relaxation times, were performed on $\mathrm{Mo}_{3} \mathrm{P}$ powder in two external magnetic fields, 0.503 and $7.067 \mathrm{~T}$. This allowed us to compare the NMR parameters in the superconducting and the normal state, respectively. The NMR signals were monitored by means of standard spin-echo sequences, consisting of $\pi / 2$ and $\pi$ pulses of 2.7 and $5.4 \mu$ s, with recycling delays ranging from 0.25 to $20 \mathrm{~s}$, in a temperature range 1.95 to $295 \mathrm{~K}$. The line shapes were obtained via fast Fourier transform (FFT) of the echo signal. Spin-lattice relaxation times $T_{1}$ were measured via the inversion recovery method, using a $\pi-\pi / 2-\pi$ pulse sequence.

The electronic structure of $\mathrm{Mo}_{3} \mathrm{P}$ was calculated by using the full-potential linearized augmented plane-wave (LAPW) method, as implemented in the WIEN2k package [26]. In particular, we employed the generalized gradient approximation (GGA) [27]. The Brillouin zone integration was performed on a regular mesh of $10 \times 10 \times 10$ points. Muffin-tin radii $\left(r_{\mathrm{mt}}\right)$ of 2.41 and $2.06 r_{\mathrm{Bohr}}$ were chosen for Mo and $\mathrm{P}$ atoms, respectively. The largest plane-wave vector was defined by $r_{\mathrm{mt}} k_{\max }=8$. The SOC was treated by using a second-order variational procedure [26].

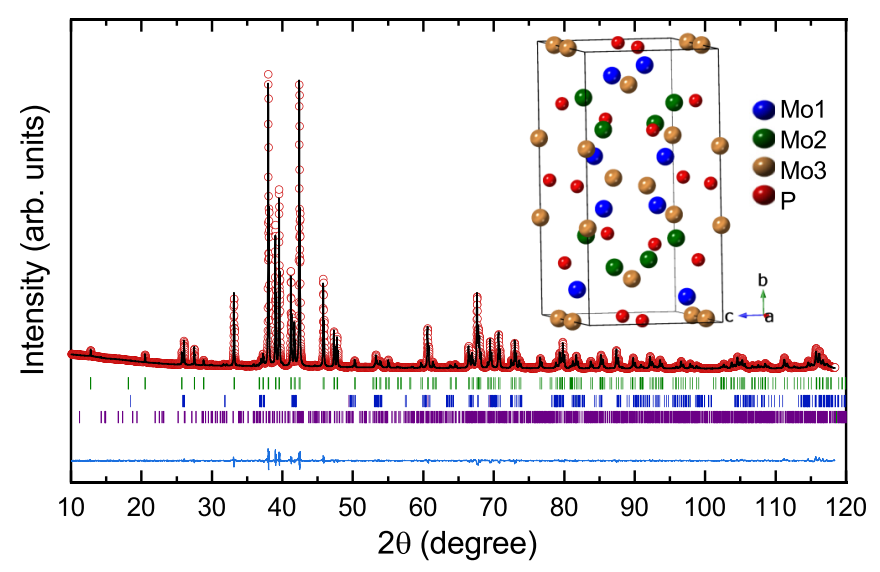

FIG. 1. Room-temperature x-ray powder diffraction pattern and Rietveld refinement for $\mathrm{Mo}_{3} \mathrm{P}$. The open red circles and the solid black line represent the experimental pattern and the Rietveldrefinement profile, respectively. The blue line at the bottom shows the residuals, i.e., the difference between calculated and experimental data. The vertical bars mark the calculated Bragg-peak positions for $\mathrm{Mo}_{3} \mathrm{P}$ (green), $\mathrm{MoO}_{2}$ (blue), and $\mathrm{Mo}_{3} \mathrm{P}_{4}$ (purple). The crystal structure (unit cell) is shown in the inset.

\section{RESULTS AND DISCUSSION}

\section{A. Crystal structure}

The crystal structure and the purity of $\mathrm{Mo}_{3} \mathrm{P}$ polycrystalline samples were checked via XRD at room temperature. Figure 1 shows a representative XRD pattern, analyzed by means of the FullProf Rietveld-analysis suite [28]. As previously reported [23], we confirm that $\mathrm{Mo}_{3} \mathrm{P}$ crystallizes in the tetragonal noncentrosymmetric $\alpha-\mathrm{V}_{3} \mathrm{~S}$-type structure with space group $I \overline{4} 2 m$ (No. 121). The refined lattice parameters, $a=b=9.79094(2) \AA$ and $c=4.826099(13) \AA$, are consistent with the reported values, but here established with a substantially improved accuracy. According to the refinements in Fig. 1, tiny amounts of $\mathrm{Mo}_{4} \mathrm{P}_{3}(3.46 \%)$ and $\mathrm{MoO}_{2}$ (4.28\%) phases were also identified. The refined $\mathrm{Mo}_{3} \mathrm{P}$ crystal structure, shown in the inset, comprises three different Mo sites and a single $\mathrm{P}$ site in the unit cell. Table I summarizes the atomic positions and the lattice parameters.

\section{B. Electrical resistivity}

The temperature-dependent electrical resistivity $\rho(T)$ of $\mathrm{Mo}_{3} \mathrm{P}$ was measured from room temperature down to $2 \mathrm{~K}$. As shown in Fig. 2, the resistivity data reveal a metallic behavior down to the superconducting transition at $T_{c}$. No anomalies associated with structural, magnetic, or charge-density-wave transitions could be detected. The electrical resistivity in the low-temperature region is shown in the inset, where the superconducting transition (with $T_{c}^{\text {onset }}=5.7 \mathrm{~K}$ and $T_{c}^{\text {zero }}=5.5 \mathrm{~K}$ ) is clearly seen. The $\rho(T)$ curve below $300 \mathrm{~K}$ can be described by the Bloch-Grüneisen-Mott (BGM) formula [29,30]:

$$
\rho(T)=\rho_{0}+4 A\left(\frac{T}{\Theta_{\mathrm{D}}^{\mathrm{R}}}\right)^{5} \int_{0}^{\frac{\Theta_{\mathrm{P}}^{\mathrm{R}}}{T}} \frac{z^{2} d z}{\left(e^{z}-1\right)\left(1-e^{-z}\right)}-\alpha T^{3} .
$$


TABLE I. Refined $\mathrm{Mo}_{3} \mathrm{P}$ crystal-lattice parameters and atomic coordinates, as determined at room temperature. $Z$ represents the number of formula units in the unit cell.

\begin{tabular}{|c|c|c|c|c|c|}
\hline \multicolumn{4}{|c|}{ Parameter } & \multicolumn{2}{|c|}{ Value } \\
\hline \multirow{2}{*}{\multicolumn{4}{|c|}{$\begin{array}{l}\text { Structure } \\
\text { Space group }\end{array}$}} & \multicolumn{2}{|c|}{ tetragonal, $\alpha-\mathrm{V}_{3} \mathrm{~S}$ type } \\
\hline & oup & & & \multicolumn{2}{|c|}{$I \overline{4} 2 m($ No. 121$)$} \\
\hline \multicolumn{4}{|l|}{$Z$} & \multicolumn{2}{|c|}{8} \\
\hline$a(\AA)$ & & & & \multicolumn{2}{|c|}{$9.79094(2)$} \\
\hline$c(\AA)$ & & & & \multicolumn{2}{|c|}{$4.826099(13)$} \\
\hline$V_{\text {cell }}(\AA$ & & & & \multicolumn{2}{|c|}{$462.6419(19)$} \\
\hline$R_{\mathrm{p}}$ & & & & \multicolumn{2}{|c|}{$2.65 \%$} \\
\hline$R_{\mathrm{wp}}$ & & & & \multicolumn{2}{|c|}{$3.70 \%$} \\
\hline$R_{\exp }$ & & & & \multicolumn{2}{|c|}{$1.14 \%$} \\
\hline$\chi_{r}^{2}$ & & & & \multicolumn{2}{|c|}{10.5} \\
\hline \multicolumn{6}{|c|}{ Atomic Coordinates } \\
\hline Atom & Wyckoff & Occ. & $x$ & $y$ & $z$ \\
\hline $\mathrm{P}$ & $8 f$ & 0.5 & $0.2924(3)$ & 0 & 0 \\
\hline $\mathrm{Mo}_{1}$ & $8 g$ & 0.5 & $0.35541(9)$ & 0 & 0.5 \\
\hline $\mathrm{Mo}_{2}$ & $8 i$ & 0.5 & $0.09289(7)$ & $0.09289(7)$ & $0.2657(3)$ \\
\hline $\mathrm{Mo}_{3}$ & $8 i$ & 0.5 & $0.29871(7)$ & $0.29871(7)$ & $0.2649(3)$ \\
\hline
\end{tabular}

Here the first term $\rho_{0}$ is the residual resistivity due to the scattering of conduction electrons on the static defects of the crystal lattice, while the second term describes the electronphonon scattering, with $\Theta_{\mathrm{D}}^{\mathrm{R}}$ being the characteristic (Debye) temperature and $A$ a coupling constant. The third term represents a contribution due to $s$ - $d$ interband scattering, $\alpha$ being the Mott coefficient [31,32]. The fit in Fig. 2 (gray line) results in $\rho_{0}=5.92(5) \mu \Omega \mathrm{cm}, A=300(7) \mu \Omega \mathrm{cm}, \Theta_{\mathrm{D}}^{\mathrm{R}}=$ $251(3) \mathrm{K}$, and $\alpha=3.4(1) \times 10^{-6} \mu \Omega \mathrm{cm} \mathrm{K}^{-3}$. The fairly large residual resistivity ratio (RRR), i.e., $\rho(300 \mathrm{~K}) / \rho_{0} \sim 45$, and the sharp superconducting transition $(\Delta T=0.2 \mathrm{~K})$ both indicate a good sample quality.

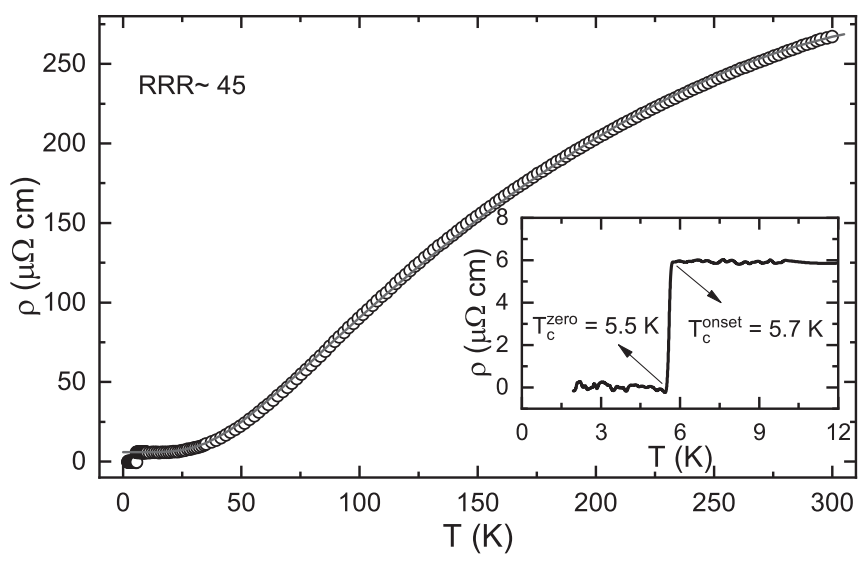

FIG. 2. Temperature dependence of the $\mathrm{Mo}_{3} \mathrm{P}$ electrical resistivity. The solid gray line through the data is a fit to Eq. (1). The inset shows the enlarged low-temperature data region, highlighting the superconducting transition.

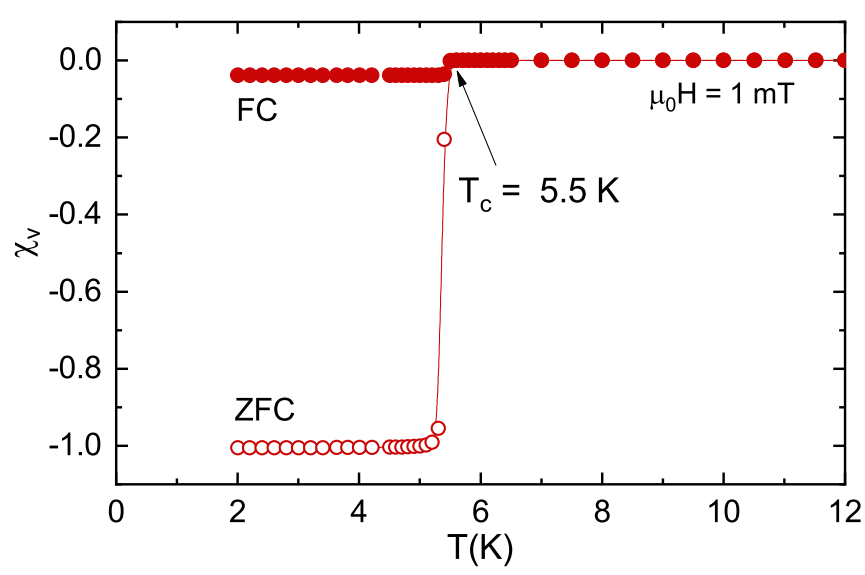

FIG. 3. Temperature dependence of the $\mathrm{Mo}_{3} \mathrm{P}$ magnetic susceptibility $\chi_{\mathrm{v}}(\equiv \chi)$, measured using ZFC and FC protocols. Data were collected in a $1 \mathrm{mT}$ applied field in a $1.8-12 \mathrm{~K}$ temperature range.

\section{Magnetic susceptibility}

The superconductivity of $\mathrm{Mo}_{3} \mathrm{P}$ was also probed by magnetic susceptibility measurements. The temperature dependence of the magnetic susceptibility $\chi(T)$ was determined using both field-cooled (FC) and zero-field-cooled (ZFC) protocols in an applied field of $1 \mathrm{mT}$. As shown in Fig. 3, $\chi(T)$ data show a superconducting transition at $T_{c}=5.5 \mathrm{~K}$, consistent with the values determined from electrical resistivity (Fig. 2) and heat capacity (see below). The splitting of the FC and ZFC susceptibilities is a typical feature of typeII superconductors with moderate to strong pinning, where the magnetic-field flux is pinned upon cooling the material in an applied field. Below $T_{c}$, a ZFC-susceptibility value of $\chi_{\mathrm{v}} \sim-1$ (after accounting for demagnetization factor, powder compactness, etc.) indicates bulk superconductivity.

\section{Lower and upper critical fields}

To determine the lower critical field $\mu_{0} H_{c 1}$ of $\mathrm{Mo}_{3} \mathrm{P}$, its field-dependent magnetization $M(H)$ was measured at various temperatures up to $T_{c}$, as shown in Fig. 4(a). The $M(H)$ curves, recorded using a ZFC-protocol, show the typical response of a type-II superconductor. The estimated $\mu_{0} H_{c 1}$ values at different temperatures, determined from the deviation of $M(H)$ from linearity, are plotted in Fig. 4(b). The solid line is a fit to $\mu_{0} H_{c 1}(T)=\mu_{0} H_{c 1}(0)\left[1-\left(T / T_{c}\right)^{2}\right]$. This provides a lower critical field $\mu_{0} H_{c 1}(0)=29.4(2) \mathrm{mT}$, consistent with $24.9(5) \mathrm{mT}$, the value calculated from the magnetic penetration depth $\lambda_{0}$ (see below).

To investigate the behavior of the upper critical field $\mu_{0} H_{c 2}$, we measured the temperature-dependent electrical resistivity $\rho(T)$ and specific heat $C(T) / T$ at various applied magnetic fields, as well as the field-dependent magnetization $M(H)$ at various temperatures up to $T_{c}$. As shown in Figs. 5(a) and $5(\mathrm{~b})$, the superconducting transition, determined from $\rho(T)$ and specific-heat $C(T) / T$ data, shifts towards lower temperature upon increasing the magnetic field. In zero magnetic field, $T_{c}=5.5 \mathrm{~K}$ determined from $C(T) / T$ is consistent with the $T_{c}$ values determined from $\rho(T)$ (Fig. 2) and $\chi(T)$ (Fig. 3) data. In the latter case, as shown in Fig. 5(c), the diamagnetic signal disappears once the applied magnetic 

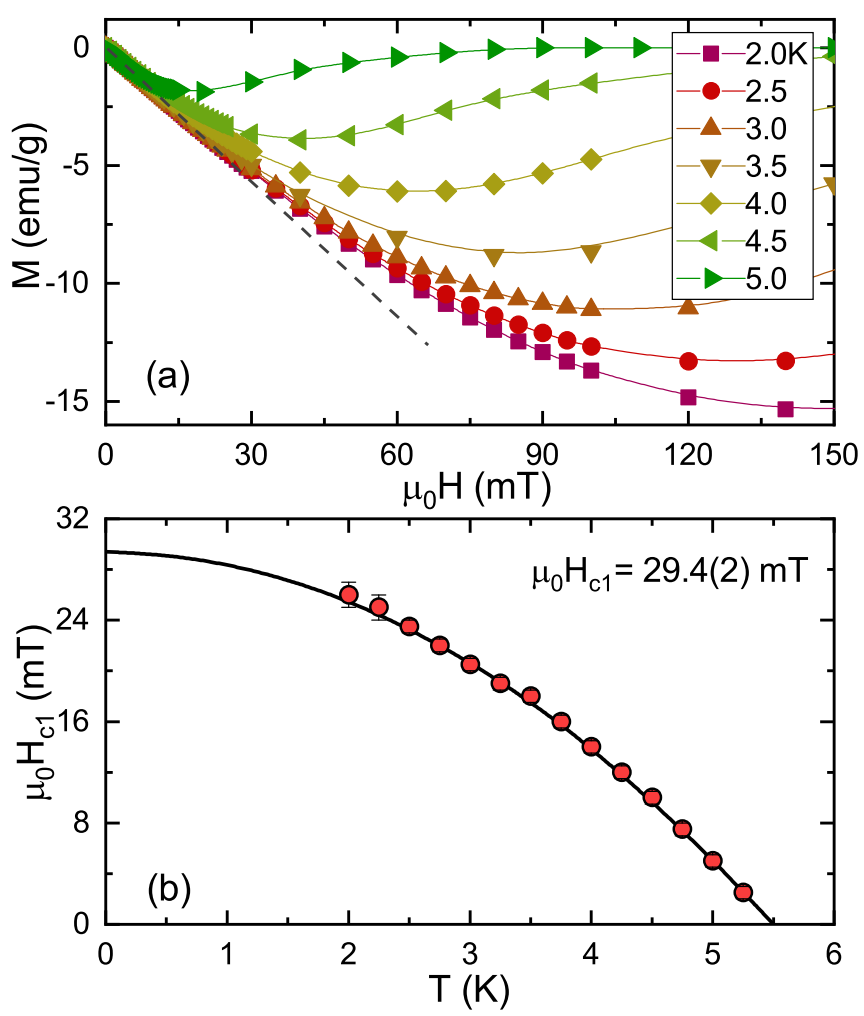

FIG. 4. (a) Field-dependent magnetization $M(H)$ recorded at various temperatures up to $T_{c}$. For each temperature, the lower critical field $\mu_{0} H_{c 1}$ was determined as the value at which $M(H)$ deviates from linearity (dashed line). (b) Estimated lower critical field $\mu_{0} H_{c 1}$ as a function of temperature, with the solid line representing a fit to $\mu_{0} H_{c 1}(T)=\mu_{0} H_{c 1}(0)\left[1-\left(T / T_{c}\right)^{2}\right]$.

field exceeds the upper critical field. Figure 5(d) summarizes the upper critical fields vs the superconducting transition temperatures $T_{c}$, as derived from $\rho(T, H), C(T, H) / T$, and $M(H, T)$ data, respectively. The temperature dependence of $\mu_{0} H_{c 2}(T)$ was analyzed by means of the semiempirical model $\mu_{0} H_{c 2}(T)=\mu_{0} H_{c 2}(0)\left(1-t^{2}\right) /\left(1+t^{2}\right)$, where $t=T / T_{c}$ is the normalized temperature (see, e.g., [33]). This model follows from the Ginzburg-Landau (GL) relation between $H_{c 2}$ and the coherence length $\xi$ (see below), by assuming $\xi(t) \propto$ $\sqrt{\left(1+t^{2}\right) /\left(1-t^{2}\right)}$. Although the GL theory is strictly valid near $T_{c}$, the above form of $H_{c 2}(T)$ has been shown to be satisfied in a wider temperature range. The solid lines in Fig. 5(d) are fits to the GL model, which gives $\mu_{0} H_{c 2}^{\mathrm{GL}}(0)=$ 0.94(1) $\mathrm{T}$ and $1.09 \mathrm{~T}$ for specific-heat and magnetization data and for electrical-resistivity data, respectively. For a comparison, we estimated the upper critical field also by means of the Werthamer-Helfand-Hohenberg (WHH) model [34]. The dashed-lines in Fig. 5(d) are fits to a WHH model without spin-orbit scattering and give $\mu_{0} H_{c 2}^{\mathrm{WHH}}(0)=0.76(1) \mathrm{T}$ and 0.83 (1) $\mathrm{T}$ for specific-heat and magnetization data and for electrical-resistivity data, respectively.

At low fields, both GL and WHH models describe the experimental data very well. At higher applied fields, however, the WHH-type fits deviate significantly from the data, clearly providing underestimated critical-field values. The remarkable agreement of the less elaborate GL model with ex-

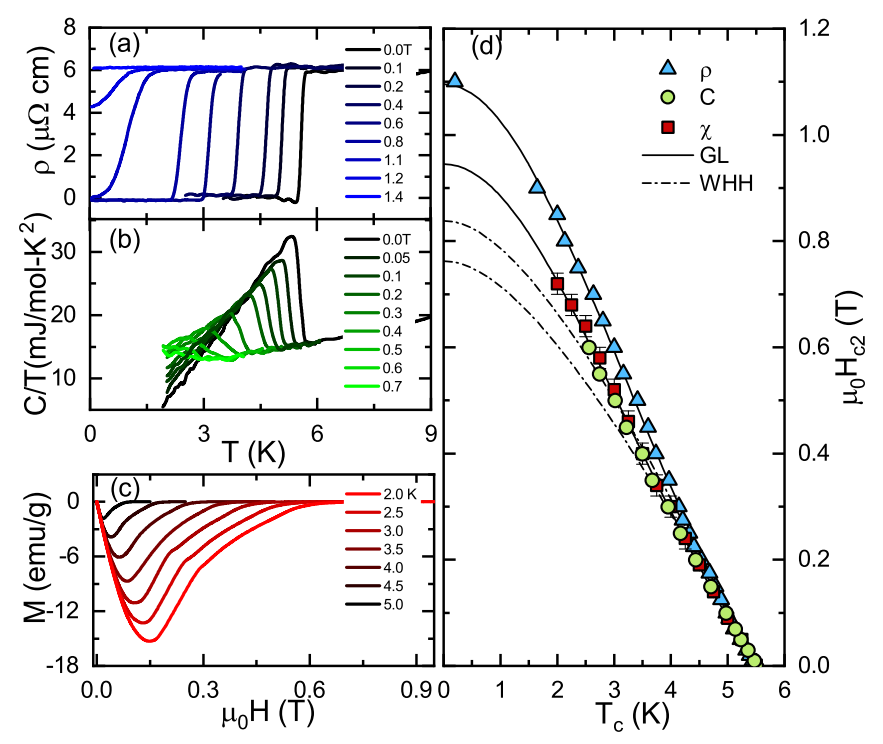

FIG. 5. Temperature-dependent electrical resistivity $\rho(T, H)$ (a) and specific heat $C / T(T, H)$ (b) for different applied magnetic fields, up to $1.4 \mathrm{~T}$. In the first case, $T_{c}$ was defined as the onset of zero resistance. (c) Field-dependent magnetization $M(H, T)$ measured at various temperatures and in applied magnetic fields up to $1.0 \mathrm{~T}$. From $\rho(T, H), C(T, H) / T$, and $M(H, T)$ one can derive the upper critical field $\mu_{0} H_{c 2}$, as shown in (d). The solid lines represent fits to an effective GL model, whereas the dash-dotted lines are fits based on a WHH model without spin-orbit scattering.

perimental data is clearly seen in Fig. 5(d). We note that while the specific-heat, magnetization, and electrical-resistivity data sets agree well at low fields $(<0.25 \mathrm{~T})$, at higher fields, the transition temperatures determined from $\rho(T, H)$ are systematically higher than those derived from $C(T, H) / T$ and $M(H, T)$ data. A similar behavior has also been found in other NCSCs, e.g., LaPtSi, BiPd, or $\mathrm{LaTSi}_{3}(T=\mathrm{Pd}, \mathrm{Pt}$, and Ir) [35-39], as well as in standard superconductors. Generally, the surface/filamentary superconductivity above bulk $T_{c}$, or a strong anisotropy of the upper critical field, is proposed as the cause of the differing $T_{c}$ values. In order to clarify this issue, studies of single-crystal specimens are highly desirable.

In the GL theory of superconductivity, the coherence length $\xi$ can be calculated from $\xi=\sqrt{\Phi_{0} / 2 \pi H_{c 2}}$, where $\Phi_{0}=$ $2.07 \times 10^{-3} \mathrm{~T} \mu \mathrm{m}^{2}$ is the quantum of magnetic flux. With a bulk $\mu_{0} H_{c 2}(0)=0.94(1) \mathrm{T}$, the calculated $\xi(0)$ is $18.7(1) \mathrm{nm}$. The magnetic penetration depth $\lambda$ is related to the coherence length $\xi$ and the lower critical field $\mu_{0} H_{c 1}$ via $\mu_{0} H_{c 1}=$ $\left(\Phi_{0} / 4 \pi \lambda^{2}\right)[\ln (\kappa)+0.5]$, where $\kappa=\lambda / \xi$ is the GL parameter [40]. By using $\mu_{0} H_{c 1}=29.4 \mathrm{mT}$ and $\xi=18.7 \mathrm{~nm}$, the resulting magnetic penetration depth $\lambda_{\mathrm{GL}}=113(1) \mathrm{nm}$ is consistent with 126(1) (zero-field extrapolation) and 121(2) $\mathrm{nm}(50 \mathrm{mT})$, the experimental values from $\mathrm{TF}-\mu \mathrm{SR}$ data (see Sec. III F and Table II). A GL parameter $\kappa \sim 7$, almost ten times larger than the $1 / \sqrt{2}$ threshold value, clearly confirms that $\mathrm{Mo}_{3} \mathrm{P}$ is a type-II superconductor.

\section{E. Zero-field specific heat}

Specific-heat data offer valuable insight into the superconducting properties, including the gap value and its symmetry. 
TABLE II. Normal- and superconducting-state properties of $\mathrm{Mo}_{3} \mathrm{P}$, as determined from electrical-resistivity, magneticsusceptibility, specific-heat, NMR, and $\mu$ SR measurements. The London penetration depth $\lambda_{\mathrm{L}}$, effective mass $m^{\star}$, bare band-structure effective mass $m_{\text {band }}^{\star}$, carrier density $n_{\mathrm{s}}$, BCS coherence length $\xi_{0}$, electronic mean-free path $l_{e}$, Fermi velocity $v_{\mathrm{F}}$, and effective Fermi temperature $T_{\mathrm{F}}$ were estimated following Eqs. (40)-(50) in Ref. [65].

\begin{tabular}{|c|c|c|}
\hline Property & Unit & Value \\
\hline$T_{c}^{\mathrm{a}}$ & $\mathrm{K}$ & $5.5(2)$ \\
\hline$\rho_{0}$ & $\mu \Omega \mathrm{cm}$ & $5.92(5)$ \\
\hline$\Theta_{D}^{R}$ & $\mathrm{~K}$ & $251(2)$ \\
\hline$\mu_{0} H_{c 1}$ & $\mathrm{mT}$ & $29.4(2)$ \\
\hline$\mu_{0} H_{c 1}^{\mu \mathrm{SR}}$ & $\mathrm{mT}$ & $24.9(5)$ \\
\hline$\mu_{0} H_{c 2}^{\rho}$ & $\mathrm{T}$ & $1.09(1)$ \\
\hline$\mu_{0} H_{c 2}^{\chi, C}$ & $\mathrm{~T}$ & $0.94(1)$ \\
\hline$\mu_{0} H_{c 2}^{\mu \mathrm{SR}}$ & $\mathrm{T}$ & $0.96(3)$ \\
\hline$\xi(0)$ & $\mathrm{nm}$ & $18.7(1)$ \\
\hline$\gamma_{n}$ & $\mathrm{~mJ} /\left(\mathrm{mol} \mathrm{K}^{2}\right)$ & $10.3(4)$ \\
\hline$\Theta_{\mathrm{D}}^{\mathrm{C}}$ & $\mathrm{K}$ & $225(3)$ \\
\hline$\lambda_{\text {ep }}$ & & $0.72(1)$ \\
\hline$N\left(\epsilon_{\mathrm{F}}\right)$ & states/(eV f.u.) & $2.2(1)$ \\
\hline$N_{\text {band }}\left(\epsilon_{\mathrm{F}}\right)$ & states/(eV f.u.) & $1.28(1)$ \\
\hline$N\left(\epsilon_{\mathrm{F}}\right)^{\mathrm{DFT}}$ & states/(eV f.u.) & 1.56 \\
\hline$E_{\mathrm{ASOC}}$ & $\mathrm{meV}$ & 90 \\
\hline$\Delta_{0}^{\mu \mathrm{SR}}$ & $\mathrm{meV}$ & $0.83(1)$ \\
\hline$\Delta_{0}^{C}$ & $\mathrm{meV}$ & $0.82(1)$ \\
\hline$\Delta C / \gamma_{\mathrm{n}} T_{c}$ & & $1.44(1)$ \\
\hline$\lambda_{0}{ }^{\mathrm{b}}$ & $\mathrm{nm}$ & $126(1)$ \\
\hline$\lambda_{0}$ & $\mathrm{~nm}$ & $121(2)$ \\
\hline$\lambda_{\mathrm{GL}}$ & $\mathrm{nm}$ & 113(1) \\
\hline$\lambda_{\mathrm{L}}$ & $\mathrm{nm}$ & $63(1)$ \\
\hline$m^{\star}$ & $m_{e}$ & $5.4(2)$ \\
\hline$m_{\text {band }}$ & $m_{e}$ & $3.1(2)$ \\
\hline$n_{\mathrm{s}}$ & $10^{28} \mathrm{~m}^{-3}$ & $3.86(3)$ \\
\hline$\xi_{0}$ & $\mathrm{~nm}$ & $56(1)$ \\
\hline$l_{e}$ & $\mathrm{~nm}$ & $18.8(2)$ \\
\hline$v_{\mathrm{F}}$ & $10^{5} \mathrm{~ms}^{-1}$ & $2.22(7)$ \\
\hline$T_{\mathrm{F}}$ & $10^{4} \mathrm{~K}$ & $1.78(6)$ \\
\hline
\end{tabular}

$\overline{\bar{a} \text { Similar values were determined via electrical resistivity, magnetic }}$ susceptibility, and heat-capacity measurements.

${ }^{\mathrm{b}}$ Derived from a fit to Eq. (8).

Therefore, the $\mathrm{Mo}_{3} \mathrm{P}$ specific heat was measured down to $0.1 \mathrm{~K}$ in zero field. As shown in Fig. 6, there is a clear specificheat jump at $T_{c}$, indicating a bulk superconducting transition. The electronic specific heat $C_{\mathrm{e}} / T$ was obtained by subtracting the phonon contribution from the experimental data. As shown in the inset of Fig. 6, the normal-state specific heat of $\mathrm{Mo}_{3} \mathrm{P}$ is fitted to $C / T=\gamma_{\mathrm{n}}+\beta T^{2}$, where $\gamma_{\mathrm{n}}$ is the electronic specific heat coefficient and $\beta T^{2}$ is the phonon contribution to the specific heat. The derived values are $\gamma_{\mathrm{n}}=10.3(4) \mathrm{mJ} /\left(\mathrm{mol} \mathrm{K}^{2}\right)$ and $\beta=0.67(3) \mathrm{mJ} /\left(\mathrm{mol} \mathrm{K}^{4}\right)$. The Debye temperature $\Theta_{\mathrm{D}}^{\mathrm{C}}$ can be calculated by using $\Theta_{\mathrm{D}}^{\mathrm{C}}=\left(12 \pi^{4} R n / 5 \beta\right)^{1 / 3}$, where $R=8.314 \mathrm{~J} /(\mathrm{mol} \mathrm{K})$ is the molar gas constant and $n=4$ is the number of atoms per formula unit. The resulting $\Theta_{\mathrm{D}}^{\mathrm{C}}=$ 225(3) $\mathrm{K}$ (extracted from low- $T$ data) is consistent with the value derived from electrical-resistivity data (see Fig. 2). The density of states (DOS) at the Fermi level $N\left(\epsilon_{\mathrm{F}}\right)$ was evaluated from the expression $N\left(\epsilon_{\mathrm{F}}\right)=3 \gamma_{\mathrm{n}} /\left(2 \pi^{2} k_{\mathrm{B}}^{2}\right)=2.2(1)$

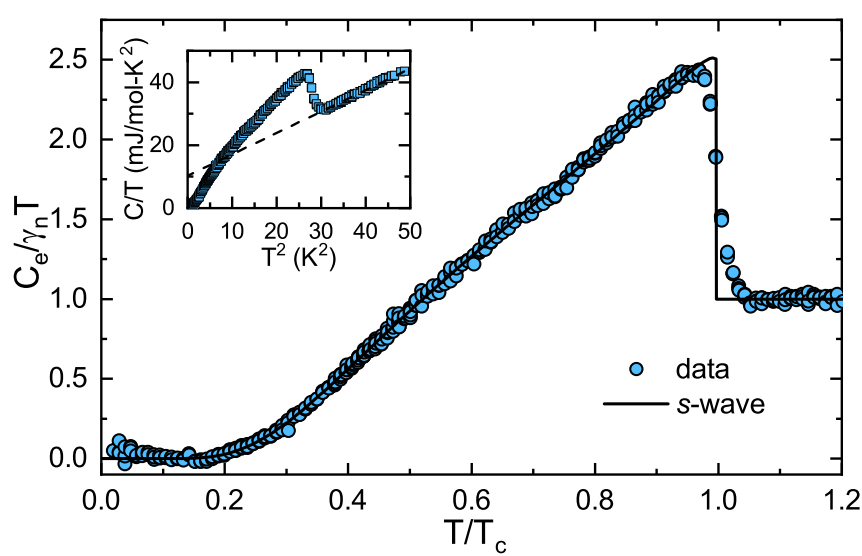

FIG. 6. Normalized electronic specific heat $C_{\mathrm{e}} / \gamma_{n} T$ for $\mathrm{Mo}_{3} \mathrm{P}$ versus $T / T_{c}$. Inset: The measured specific heat $C / T$ as a function of $T^{2}$. The dashed line in the inset is a fit to $C / T=\gamma+\beta T^{2}$, while the solid line in the main panel is the electronic specific heat calculated by considering a fully gapped $s$-wave model.

states/(eV f.u.) (accounting for spin degeneracy) [41], where $k_{\mathrm{B}}$ is the Boltzmann constant. The electron-phonon coupling constant $\lambda_{\text {ep }}$, a measure of the attractive interaction between electrons due to phonons, was estimated from the $\Theta_{\mathrm{D}}^{\mathrm{C}}$ and $T_{c}$ values by applying the semiempirical McMillan formula [42]:

$$
\lambda_{\text {ep }}=\frac{1.04+\mu^{\star} \ln \left(\Theta_{\mathrm{D}} / 1.45 T_{c}\right)}{\left(1-0.62 \mu^{\star}\right) \ln \left(\Theta_{\mathrm{D}} / 1.45 T_{c}\right)-1.04} .
$$

The Coulomb pseudopotential $\mu^{\star}$, usually lying in the $0.09-0.18$ range, was here fixed to 0.13 , a commonly used value for transition metals. From Eq. (2) we obtain $\lambda_{\text {ep }}=$ $0.72(1)$ for $\mathrm{Mo}_{3} \mathrm{P}$, almost twice the reported value for the elemental Mo $(0.41)[43,44]$. Finally, the band-structure density of states $N_{\text {band }}\left(\epsilon_{\mathrm{F}}\right)$ can be estimated from the relation $N_{\text {band }}\left(\epsilon_{\mathrm{F}}\right)=N\left(\epsilon_{\mathrm{F}}\right) /\left(1+\lambda_{\text {ep }}\right)$ [41], which gives $N_{\text {band }}\left(\epsilon_{\mathrm{F}}\right)=$ 1.28(1) states/(eV f.u.).

The electronic specific heat divided by the electronic specific heat coefficient, i.e., $C_{\mathrm{e}} / \gamma_{\mathrm{n}} T$, is shown in the main panel of Fig. 6 as a function of the reduced temperature. The temperature-independent behavior of $C_{\mathrm{e}} / \gamma_{\mathrm{n}} T$ at low $T$ suggests a fully gapped superconducting state in $\mathrm{Mo}_{3} \mathrm{P}$. The temperature-dependent superconducting-phase contribution to the entropy was calculated by means of [45]

$$
S(T)=-\frac{6 \gamma_{\mathrm{n}}}{\pi^{2} k_{\mathrm{B}}} \int_{0}^{\infty}[f \ln f+(1-f) \ln (1-f)] d \epsilon,
$$

where $f=\left(1+e^{E / k_{\mathrm{B}} T}\right)^{-1}$ is the Fermi function, $\Delta_{0}$ is the SC gap value at $0 \mathrm{~K}$, and $E(\epsilon)=\sqrt{\epsilon^{2}+\Delta^{2}(T)}$ is the excitation energy of quasiparticles, with $\epsilon$ the electron energies measured relative to the chemical potential (Fermi energy) $[45,46] . \Delta(T)$ is the same as in Sec. IIIF. The temperature-dependent electronic specific heat in the superconducting state can be calculated from $C_{\mathrm{e}}=T \frac{d S}{d T}$. The solid line in Fig. 6 represents a fit with a fully gapped $s$-wave model. The resulting superconducting gap $\Delta_{0}=0.82(1) \mathrm{meV}$ is consistent with the $\mu \mathrm{SR}$ results (see Fig. 9). The $\mathrm{Mo}_{3} \mathrm{P}$ gap value $\Delta_{0}$, as derived from both specific-heat and TF- $\mu$ SR data, is comparable to the expected weak-coupling BCS value $0.80 \mathrm{meV}$, thus indicating weakly coupled superconducting 


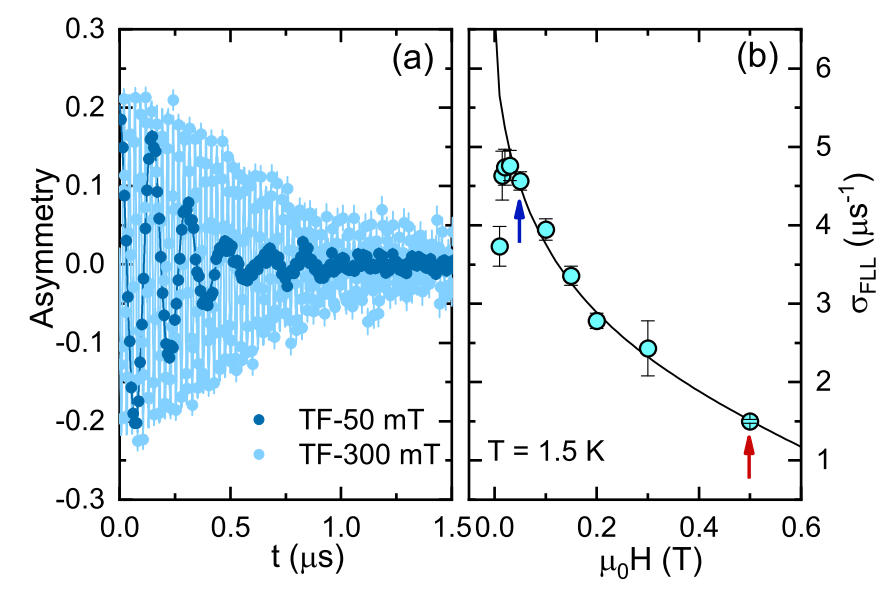

FIG. 7. (a) Time-domain TF- $\mu$ SR spectra of $\mathrm{Mo}_{3} \mathrm{P}$ measured in its superconducting state (at $T=1.5 \mathrm{~K}$ ) at 50 and $300 \mathrm{mT}$. (b) Fielddependent Gaussian relaxation rate $\sigma_{\mathrm{FLL}}(H)$. The arrows indicate the field values (50 and $500 \mathrm{mT}$ ) used in the temperature-dependent TF- $\mu$ SR studies. The solid line is a fit to Eq. (8).

pairs in $\mathrm{Mo}_{3} \mathrm{P}$. Furthermore, also the specific-heat discontinuity at $T_{c}$, i.e., $\Delta C / \gamma_{\mathrm{n}} T_{c}=1.44$, is fully consistent with the conventional BCS value of 1.43 .

\section{F. Transverse-field $\mu \mathrm{SR}$}

To investigate the superconducting properties of $\mathrm{Mo}_{3} \mathrm{P}$ at a microscopic level, we carried out $\mu \mathrm{SR}$ measurements in applied transverse fields (TFs). The optimal field value for such experiments was determined via a preliminary fielddependent $\mu \mathrm{SR}$ depolarization-rate measurement at $1.5 \mathrm{~K}$. To track the additional field-distribution broadening due to the flux-line lattice (FLL) in the mixed superconducting state, a magnetic field (up to $500 \mathrm{mT}$ ) was applied in the normal state and then the sample was cooled down to $1.5 \mathrm{~K}$. Figure 7(a) shows the TF spectra in an applied field of 50 and $300 \mathrm{mT}$, respectively. The solid lines represent fits using the same model as described in Eq. (4) below. The resulting Gaussian relaxation rate $\sigma_{\mathrm{FLL}}(H)$ is summarized in Fig. 7(b). Above the lower critical field $\mu_{0} H_{c 1}(29.4 \mathrm{mT})$, the relaxation rate decreases continuously. By considering the decrease of the intervortex distance with the field and the vortex-core effects, a field of $50 \mathrm{mT}$ was chosen for the temperature-dependent $\mathrm{TF}-\mu \mathrm{SR}$ studies. For a comparison, the $\mathrm{TF}-\mu \mathrm{SR}$ relaxation was also measured in a field of $500 \mathrm{mT}$. The field dependence of the Gaussian relaxation rate was analyzed following Eq. (8) (see details below). The derived zero-temperature $\mu_{0} H_{c 2}=0.96(1) \mathrm{T}$ and magnetic penetration depth $\lambda_{0}=126(1) \mathrm{nm}$ are consistent with the temperature-dependent $50 \mathrm{mT}$ results (see Table II).

The TF- $\mu$ SR time spectra were collected at various temperatures up to $T_{c}$, following a field-cooling protocol on both GPS and HAL instruments. Figures 8(a) and 8(b) show two representative $\mathrm{TF}-\mu \mathrm{SR}$ spectra collected above $(7.5 \mathrm{~K})$ and below $T_{c}(1.5 \mathrm{~K})$ on GPS. The enhanced depolarization rate below $T_{c}$ reflects the inhomogeneous field distribution due to the FLL, which causes the additional distribution broadening in the mixed state [see Fig. 8(c)]. The time evolution of the
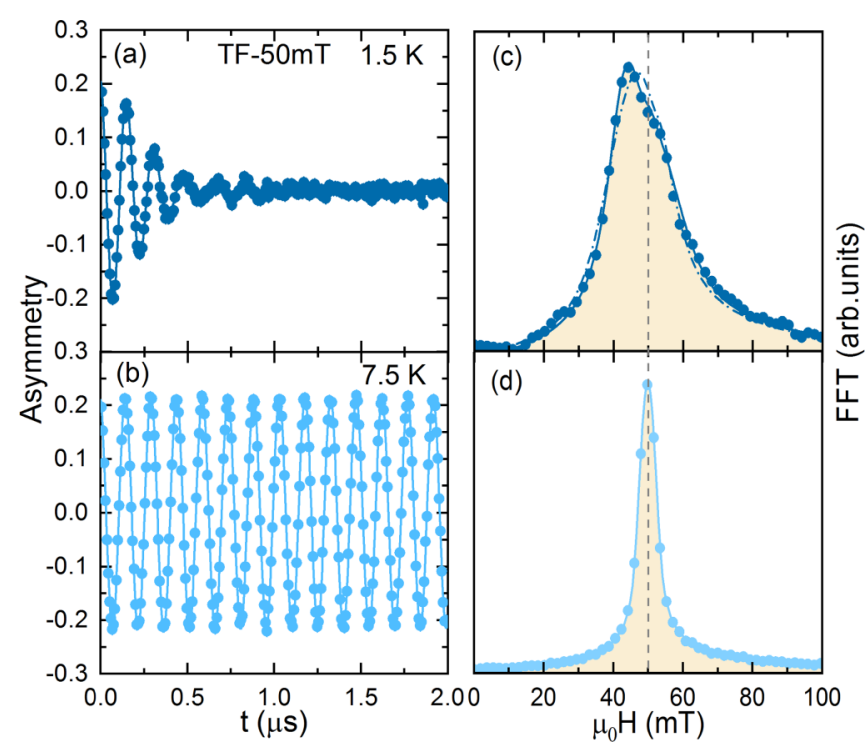

FIG. 8. TF- $\mu$ SR time-dependent spectra, collected at $1.5 \mathrm{~K}$ (a) and $7.5 \mathrm{~K}$ (b) in an applied field of $50 \mathrm{mT}$. Fourier transforms of the above time spectra at $1.5 \mathrm{~K}$ (c) and $7.5 \mathrm{~K}$ (d). The solid lines are fits to Eq. (4) using two oscillations, while the dash-dotted line in (c) represents a fit with a single oscillation. The vertical dashed line indicates the applied magnetic field. Note the clear diamagnetic shift below $T_{c}$ in (c).

$\mu$ SR asymmetry can be modeled by

$$
\begin{aligned}
A_{\mathrm{TF}}(t)= & \sum_{i=1}^{n} A_{i} \cos \left(\gamma_{\mu} B_{i} t+\phi\right) e^{-\sigma_{i}^{2} t^{2} / 2} \\
& +A_{\mathrm{bg}} \cos \left(\gamma_{\mu} B_{\mathrm{bg}} t+\phi\right)
\end{aligned}
$$

Here $A_{i}$ and $A_{\mathrm{bg}}$ represent the initial muon-spin asymmetries for muons implanted in the sample and sample holder, respectively, with the latter not undergoing any depolarization. $B_{i}$ and $B_{\mathrm{bg}}$ are the local fields sensed by implanted muons in the sample and sample holder, $\gamma_{\mu}=2 \pi \times 135.53 \mathrm{MHz} / \mathrm{T}$ is the muon gyromagnetic ratio, $\phi$ is the shared initial phase, and $\sigma_{i}$ is a Gaussian relaxation rate of the $i$ th component. The number of required components is material dependent, generally in the $1 \leqslant n \leqslant 5$ range. For superconductors with a large $\kappa(\gg 1)$ value, the magnetic penetration depth is much larger than the coherence length. Hence, the field distribution is narrow and a single-oscillating component is sufficient to describe $A(t)$. In case of a small $\kappa(\gtrsim 1 / \sqrt{2})$ value, the magnetic penetration depth is comparable to the coherence length. This implies a broad field distribution, in turn requiring multiple oscillations to describe $A(t)$.

Figures 8(c) and 8(d) show the FFT spectra of the TF- $\mu$ SR time-domain asymmetries at $1.5 \mathrm{~K}$ and $7.5 \mathrm{~K}$. Solid lines represent fits to Eq. (4) using two oscillations (i.e., $n=2$ ) in the superconducting phase and one oscillation in the normal phase. In the $1.5 \mathrm{~K}$ case, a single-component oscillation [dashdotted line in panel (c)] is clearly unable to fit the $T<T_{c}$ data. The derived Gaussian relaxation rates as a function of temperature are summarized in the insets of Fig. 9. Above $T_{c}$ the relaxation rate is small and temperature independent, but below $T_{c}$ it starts to increase due to the onset of the FLL 


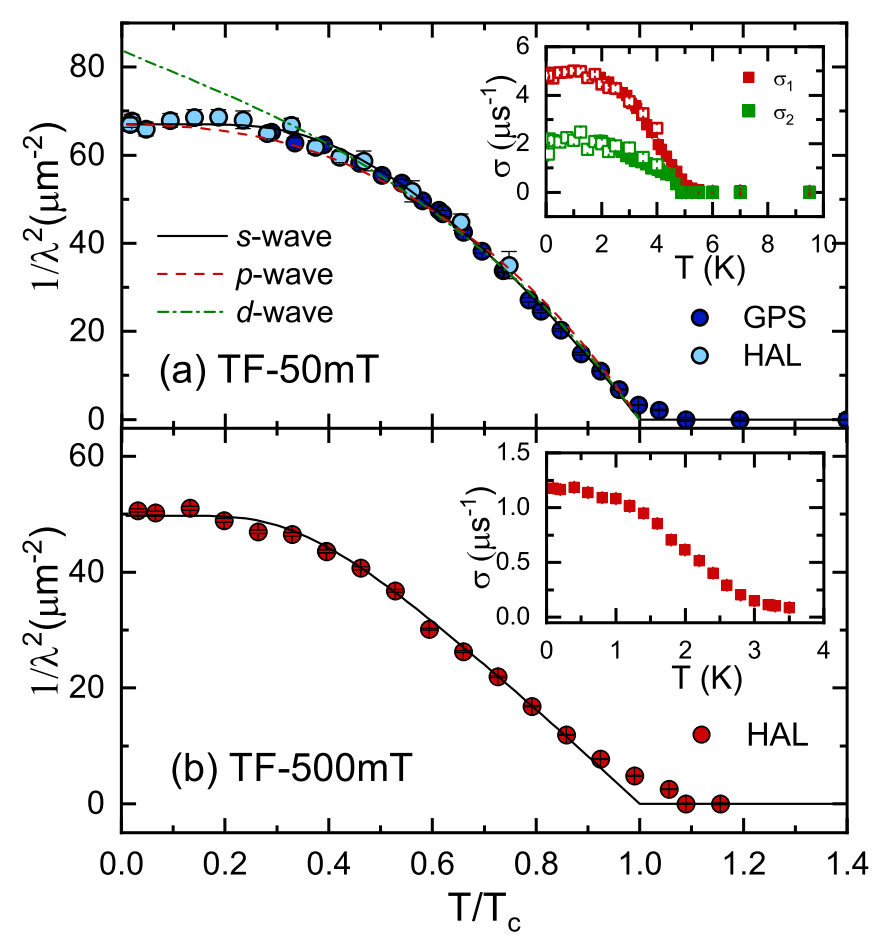

FIG. 9. Superfluid density vs temperature, as determined from TF- $\mu$ SR measurements in an applied magnetic field of $50 \mathrm{mT}$ (a) and $500 \mathrm{mT}$ (b). The insets show the temperature dependence of the muon-spin relaxation rate $\sigma(T)$. Two components are required to describe the $50 \mathrm{mT}$ experimental data [see details in Fig. 8(c)]. The different lines represent fits to various models, including $s-, p$-, and $d$-wave pairing (see text for details).

and the increase in superfluid density. Unlike the TF- $\mu$ SR spectra collected at $50 \mathrm{mT}$, the $500 \mathrm{mT}$ data sets can be described by a single oscillation. This reflects the narrower internal-field distribution for applied fields comparable to $H_{c 2}$. In case of multicomponent oscillations (at $50 \mathrm{mT}$ ), the firstterm in Eq. (4) describes the field distribution as the sum of $n$ Gaussian relaxations [47]:

$$
P(B)=\gamma_{\mu} \sum_{i=1}^{2} \frac{A_{i}}{\sigma_{i}} \exp \left[-\frac{\gamma_{\mu}^{2}\left(B-B_{i}\right)^{2}}{2 \sigma_{i}^{2}}\right] .
$$

Then, the first and second moments of the field distribution in the sample were calculated by

$$
\begin{aligned}
\langle B\rangle & =\sum_{i=1}^{2} \frac{A_{i} B_{i}}{A_{\mathrm{tot}}}, \\
\left\langle B^{2}\right\rangle & =\frac{\sigma_{\mathrm{eff}}^{2}}{\gamma_{\mu}^{2}}=\sum_{i=1}^{2} \frac{A_{i}}{A_{\mathrm{tot}}}\left[\frac{\sigma_{i}^{2}}{\gamma_{\mu}^{2}}-\left(B_{i}-\langle B\rangle\right)^{2}\right],
\end{aligned}
$$

where $A_{\mathrm{tot}}=\sum_{i=1}^{2} A_{i}$. In the superconducting state, the measured Gaussian relaxation rate includes contributions from both a temperature-independent relaxation due to nuclear moments $\left(\sigma_{\mathrm{n}}\right)$ and the FLL $\left(\sigma_{\mathrm{FLL}}\right)$. The FLL-related relaxation can be extracted by subtracting the nuclear contribution according to $\sigma_{\mathrm{FLL}}=\sqrt{\sigma_{\mathrm{eff}}^{2}-\sigma_{\mathrm{n}}^{2}}$. Since $\sigma_{\mathrm{FLL}}$ is directly related to the magnetic penetration depth and the superfluid density
$\left(\sigma_{\mathrm{FLL}} \propto 1 / \lambda^{2}\right)$, the superconducting gap value and its symmetry can be determined from the measured $\sigma_{\mathrm{FLL}}(T)$.

For small applied magnetic fields $\left(H_{\text {appl }} / H_{c 2} \ll 1\right)$ and large $\kappa$ values $(\kappa \gg 1), \sigma_{\mathrm{FLL}}$ is field independent and proportional to $\lambda^{-2}[40,48]$. In the $\mathrm{Mo}_{3} \mathrm{P}$ case, however, the upper critical field $\mu H_{c 2}$ is relatively small $(0.94 \mathrm{~T})$ compared to the applied TF field (50 and $500 \mathrm{mT}$ ). Therefore, to extract the penetration depth from the measured $\sigma_{\mathrm{FLL}}$, the effects of overlapping vortex cores with increasing field have to be considered, requiring the following expression for calculating the magnetic penetration depth $\lambda[40,48]$ :

$$
\sigma_{\mathrm{FLL}}=0.172 \frac{\gamma_{\mu} \Phi_{0}}{2 \pi}(1-h)\left[1+1.21(1-\sqrt{h})^{3}\right] \lambda^{-2},
$$

where $h=H_{\text {appl }} / H_{\mathrm{c} 2}$, with $H_{\text {appl }}$ the applied magnetic field. The above expression is valid for type-II superconductors with $\kappa \geqslant 5$ in the $0.25 / \kappa^{1.3} \lesssim h \leqslant 1$ field range. With $\kappa \sim 6$ and $h=0.053$ (TF $50 \mathrm{mT}$ ) and 0.53 (TF $500 \mathrm{mT}$ ), $\mathrm{Mo}_{3} \mathrm{P}$ fulfills the above conditions.

By using Eq. (8), we could calculate the inverse square of the magnetic penetration depth, whose temperature dependence is shown in Fig. 9. To gain insight into the superconducting pairing symmetry in $\mathrm{Mo}_{3} \mathrm{P}$, the temperaturedependent superfluid density $\rho_{\mathrm{sc}}(T)\left[\lambda^{-2}(T) \propto \rho_{s}(T)\right]$ was further analyzed by means of different models, generally described by

$$
\rho_{\mathrm{sc}}(T)=1+2\left\langle\int_{\Delta_{k}}^{\infty} \frac{E}{\sqrt{E^{2}-\Delta_{k}^{2}}} \frac{\partial f}{\partial E} d E\right\rangle_{\mathrm{FS}},
$$

where $\Delta_{k}$ is an angle-dependent gap function, $f=(1+$ $\left.e^{E / k_{\mathrm{B}} T}\right)^{-1}$ is the Fermi function, and $\langle\cdots\rangle_{\mathrm{FS}}$ represents an average over the Fermi surface [45]. The gap function can be written as $\Delta_{\mathrm{k}}(T)=\Delta(T) g_{k}$, where $\Delta$ is the maximum gap value and $g_{\mathrm{k}}$ is the angular dependence of the gap, equal to $1, \cos 2 \psi$, and $\sin \theta$ for an $s-, d$-, and $p$-wave model, respectively. Here $\psi$ and $\theta$ are azimuthal angles. The temperature dependence of the gap is assumed to follow $\Delta(T)=$ $\Delta_{0} \tanh \left\{1.82\left[1.018\left(T_{c} / T-1\right)\right]^{0.51}\right\}$ [49], where $\Delta_{0}$, the gap value at zero temperature, is the only adjustable parameter. Note that the function $\Delta(T)$ is practically model independent.

Three different models, including $s, d$, and $p$ waves, were used to describe the temperature-dependent superfluid density $\lambda^{-2}(T)$ measured in an applied field of $50 \mathrm{mT}$ [Fig. 9(a)], with the first two being singlet pairing and the last one triplet paring. For the $s$ - and $p$-wave model, the estimated gap values are $0.83(1)$ and $1.12(1) \mathrm{meV}$, respectively, with the shared zero-temperature magnetic penetration depth $\lambda_{0}=$ 121(2) nm, while for the $d$-wave model, the estimated $\lambda_{0}$ and gap values are 109(2) nm and 1.10(1) meV. As can be seen in Fig. 9(a), the temperature-independent behavior of $\lambda^{-2}(T)$ below $1 / 3 T_{c}$ strongly suggests a nodeless superconductivity in $\mathrm{Mo}_{3} \mathrm{P}$. Therefore, $\lambda^{-2}(T)$ is clearly more consistent with a single fully gapped $s$-wave model. In case of $d$ - or $p$-wave models, a less-good agreement with the measured $\lambda^{-2}$ values is found, especially at low temperature. Such conclusions are further supported by the low- $T$ specific-heat data shown in Fig. 6. Also in the TF $500 \mathrm{mT}$ case [see Fig. 9(b)], the $\lambda^{-2}(T)$ dependence is consistent with an $s$-wave model, 


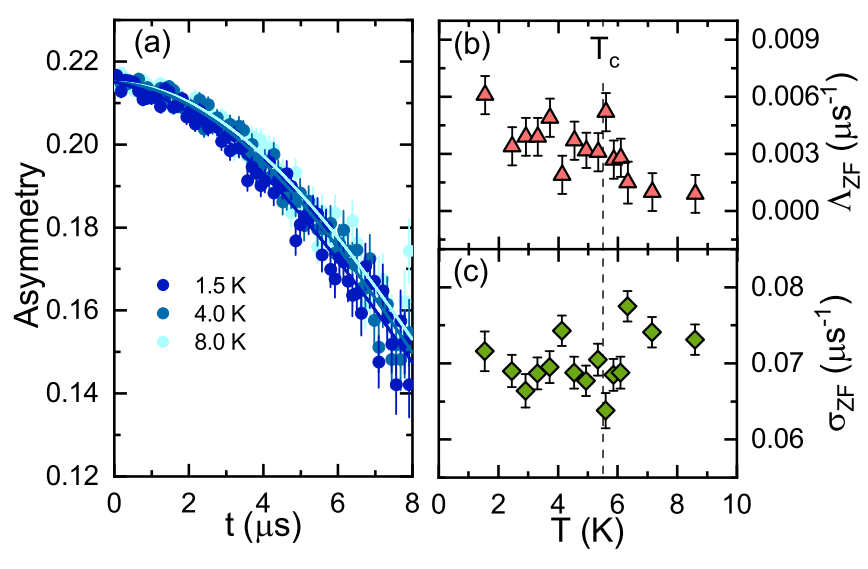

FIG. 10. (a) Representative ZF- $\mu$ SR spectra for $\mathrm{Mo}_{3} \mathrm{P}$ in the superconducting $(1.5$ and $4 \mathrm{~K})$ and the normal state $(8 \mathrm{~K})$. The solid lines are fits to Eq. (10), as described in the text. Temperature dependence of the Lorentzian relaxation rate $\Lambda_{\mathrm{ZF}}$ (b) and Gaussian relaxation rate $\sigma_{\mathrm{ZF}}(\mathrm{c})$. The dashed line, which marks the bulk $T_{c}$ at $5.5 \mathrm{~K}$, is a guide to the eyes. None of the reported fit parameters show distinct anomalies across $T_{c}$.

leading to a $\lambda_{0}=141(2) \mathrm{nm}$ and to a gap value $\Delta_{0}=$ $1.57 k_{\mathrm{B}} T_{c}=0.41(1) \mathrm{meV}$.

\section{G. Zero-field $\mu \mathrm{SR}$}

We performed also $\mathrm{ZF}-\mu \mathrm{SR}$ measurements, in order to search for a possible TRS breaking in the superconducting state of $\mathrm{Mo}_{3} \mathrm{P}$. The large muon gyromagnetic ratio, combined with the availability of $100 \%$ spin-polarized muon beams, make ZF- $\mu$ SR a very sensitive probe for detecting small spontaneous magnetic fields. This technique has been successfully used to detect the TRS breaking in the superconducting states of different types of materials $[8,11,12,15,16,50]$. Normally, in the absence of external fields, the onset of SC does not imply changes in the ZF muon-spin relaxation rate. However, if the TRS is broken, the onset of tiny spontaneous currents gives rise to associated (weak) magnetic fields, readily detected by $\mathrm{ZF}-\mu \mathrm{SR}$ as an increase in muon-spin relaxation rate. Representative $\mathrm{ZF}-\mu \mathrm{SR}$ spectra collected above $(8 \mathrm{~K})$ and below $T_{c}(1.5$ and $4 \mathrm{~K})$ are shown in Fig. 10. For nonmagnetic materials, in the absence of applied fields, the relaxation is mainly determined by the randomly oriented nuclear moments. Consequently, the $\mathrm{ZF}-\mu \mathrm{SR}$ spectra of $\mathrm{Mo}_{3} \mathrm{P}$ could be modeled by means of a combined Lorentzian and Gaussian Kubo-Toyabe relaxation function [51,52]:

$$
A_{\mathrm{ZF}}=A_{\mathrm{s}}\left[\frac{1}{3}+\frac{2}{3}\left(1-\sigma_{\mathrm{ZF}}^{2} t^{2}-\Lambda_{\mathrm{ZF}} t\right) e^{\left(-\frac{\sigma_{\mathrm{ZF}}^{2} t^{2}}{2}-\Lambda_{\mathrm{ZF}} t\right)}\right]+A_{\mathrm{bg}}
$$

Here $A_{\mathrm{s}}$ and $A_{\mathrm{bg}}$ represent the initial muon-spin asymmetries for muons implanted in the sample and sample holder, respectively. In polycrystalline samples, the 1/3-nonrelaxing and 2/3-relaxing components of the asymmetry correspond to the powder average of the local internal fields with respect to the initial muon-spin orientation. After fixing $A_{\mathrm{s}}$ to its average value of 0.215 , the resulting fit parameters are shown in Figs. 10(b) and 10(c). The weak Gaussian and Lorentzian relaxation rates reflect the absence of electronic magnetic moments and the small value of the $\mathrm{Mo}_{3} \mathrm{P}$ nuclear moments. Although the ZF- $\mu$ SR spectra in Fig. 10(a) show tiny differences between the normal and the superconducting state, the resulting $\Lambda_{\mathrm{ZF}}(T)$ and $\sigma_{\mathrm{ZF}}(T)$ parameters show no distinct changes across $T_{c}$. Note that the tiny increase of $\Lambda_{\mathrm{ZF}}$ between the normal and superconducting state [Fig. 10(b)] is not related to a TRS-breaking effect, but to the correlated decrease of $\sigma_{\mathrm{ZF}}$ [Fig. 10(c)]. The clear lack of an additional relaxation below $T_{c}$ implies that the TRS is preserved in the superconducting state of $\mathrm{Mo}_{3} \mathrm{P}$.

\section{H. ${ }^{31} \mathrm{P}$ NMR in the normal phase}

NMR is a versatile technique for investigating the electronic properties of materials, in particular, their electron correlations, complementary to $\mu \mathrm{SR}$ with respect to probe location. Considering the rather low $T_{c}$ and $H_{c 2}(0)$ values of $\mathrm{Mo}_{3} \mathrm{P}$, as well as the detailed $\mu \mathrm{SR}$ study of its superconducting properties (see previous section), we mostly employed NMR to investigate the normal-state electronic properties of $\mathrm{Mo}_{3} \mathrm{P}$. To this aim, ${ }^{31} \mathrm{P}$ NMR measurements were performed at 7 and $0.5 \mathrm{~T}$, i.e., above and below $H_{c 2}(0)(0.94 \mathrm{~T})$. For both fields, the ${ }^{31} \mathrm{P}$ NMR reference frequency $v_{0}$ was determined by using a solid-state $\mathrm{NH}_{4} \mathrm{H}_{2} \mathrm{PO}_{4}$ sample, whose Larmor frequency coincides with that of phosphoric acid. The ${ }^{31} \mathrm{P}$ NMR shifts were then calculated with respect to this reference.

Typical ${ }^{31} \mathrm{P}$ NMR line shapes are shown in Fig. 15 in the Appendix. As expected, the NMR lines at $0.5 \mathrm{~T}$ show a significant broadening and frequency shift below $T_{c}$ in the superconducting phase. The $7 \mathrm{~T}$ lines, instead, are practically temperature independent, with typical shapes reflecting the Knight shift anisotropy. The Knight shift, peak position, and linewidth were obtained using the DMFIT software [53], with the best-fit parameters being $\delta_{\text {iso }}=285 \mathrm{ppm}, \Omega=$ $35 \mathrm{kHz}$, and $\eta_{\mathrm{CS}}=0.38$. Here, $\delta_{\text {iso }}=\left(\delta_{11}+\delta_{22}+\delta_{33}\right) / 3$ is the isotropic component of the shift tensor, i.e., the average of its diagonal components in the principal-axes system (PAS), the linewidth is $\Omega=\left|\delta_{11}-\delta_{33}\right|$, and $\eta_{\mathrm{CS}}=\left(\delta_{22}-\right.$ $\left.\delta_{11}\right) /\left(\delta_{33}-\delta_{\text {iso }}\right)$. The temperature evolution of the linewidths and shifts (normalized to the field value and the reference frequency, respectively) is shown in Fig. 11. The almost temperature- and field-independent linewidths and frequencies suggest a behavior close to that expected for weakly to moderately correlated metals. The above picture changes only close to $T_{c}$, below which we detect a significant drop in frequency and increase of linewidth, reflecting the electron pairing and the development of the flux-line lattice in the superconducting phase, respectively. The temperaturedependent NMR relaxation rate $1 / T_{1}$ provides useful insight into the dynamics of conduction electrons and their degree of correlation. The $1 / T_{1}$ data, shown in Fig. 12(a) (inset), indicate an almost linear behavior with slightly different slopes in the regime above and below $T^{\star} \sim 103 \mathrm{~K}$. While the change in slope might reflect a change in the electronnuclei coupling, the linearity of $1 / T_{1}(T)$ is once more a clear indication of weak to moderate electron correlations. This conclusion is confirmed by the $1 / T_{1} T$ data shown in Fig. 12(a) (main panel), where the reported product, at a first approximation considered as constant, is $0.054 \mathrm{~s}^{-1} \mathrm{~K}^{-1}$. Compared to other metallic superconductors, such as $\mathrm{MgB}_{2}$, 


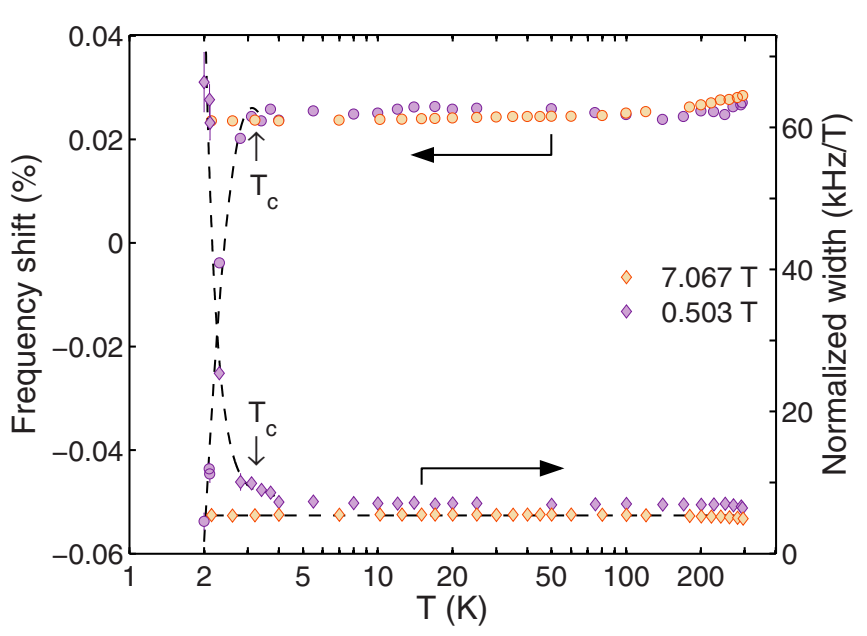

FIG. 11. Normalized ${ }^{31} \mathrm{P}$ NMR Knight shifts $\left(\delta_{\text {iso }}-v_{0}\right) / v_{0}$ (left) and linewidths (right) vs temperature, as measured at 0.5 and $7 \mathrm{~T}$. While there is a clear drop of frequency and increase of width below $T_{c}$, the data in the normal phase are practically temperature and field independent, indicating an almost ideal metallic behavior.

$\mathrm{AlB}_{2}$, and $\mathrm{ZrB}$ (see Fig. 5 in Ref. [54]), such a value indicates a good metallicity, i.e., a relatively high electron density of states at the Fermi level. Upon closer inspection we note that, after a minimum at $\sim 20 \mathrm{~K}, 1 / T_{1} T$ increases slightly with temperature. The origin of this minimum is currently unknown to us.

The Korringa relation [55] states the proportionality between the spin relaxation rate divided by the square of Knight shift (spin part) with the temperature. The total Knight shift $K$ consists of a spin $\left(K_{s}\right)$ and an orbital $\left(K_{\text {orb }}\right)$ part. Normally these can be separated by using the (typically linear) $K-\chi$ plot, with $K_{\text {orb }}$ being the intercept value to the $K$ axis. However, as shown in Fig. 16, $\mathrm{Mo}_{3} \mathrm{P}$ exhibits a complex behavior, most likely reflecting the presence of $d$-type electrons (see Appendix). In our case, considering the opposite-spin pairing of electrons in the SC phase, we can assume $K_{s}$ to be fully suppressed at $0 \mathrm{~K}$. From Fig. 11 we can determine $K_{\text {orb }} \sim$ $-0.05 \%$ and, hence, $K_{s} \sim 0.07 \%$. Originally derived for simple $s$-band metals with negligible electronic correlations, $K_{s}$ was successively extended also to $d$-band metals, where core polarization effects typically dominate both the Knight shift and the relaxation rate [56]. The experimental value of the proportionality constant and its temperature dependence provide insight into the degree of electron correlations in the normal state of a material. In particular,

$$
T_{1} T K_{s}^{2}=\alpha S_{0}, \quad \text { with } \quad S_{0}=\frac{\gamma_{e}^{2}}{\gamma_{N}^{2}} \frac{\hbar}{4 \pi k_{\mathrm{B}}},
$$

where $\gamma_{e}$ is the gyromagnetic ratio for a free electron, and $\gamma_{N}$ the gyromagnetic ratio of the probe nucleus. Here, $\alpha$ is a coefficient of correlation which, in the ideal case of uncorrelated electrons, should be 1. As shown in Fig. 12(b), in $\mathrm{Mo}_{3} \mathrm{P}$ the Korringa product is virtually temperature independent (above $T_{c}$ ), since in our case small variations in $1 / T_{1} T$ are mostly compensated by those of the Knight shift (see inset in Fig. 16). The resulting $\alpha$ value is 5.8, i.e., much higher than unity. Since the ${ }^{31} \mathrm{P}$ NMR $K_{s}$ shift is mostly due to $4 d$-type
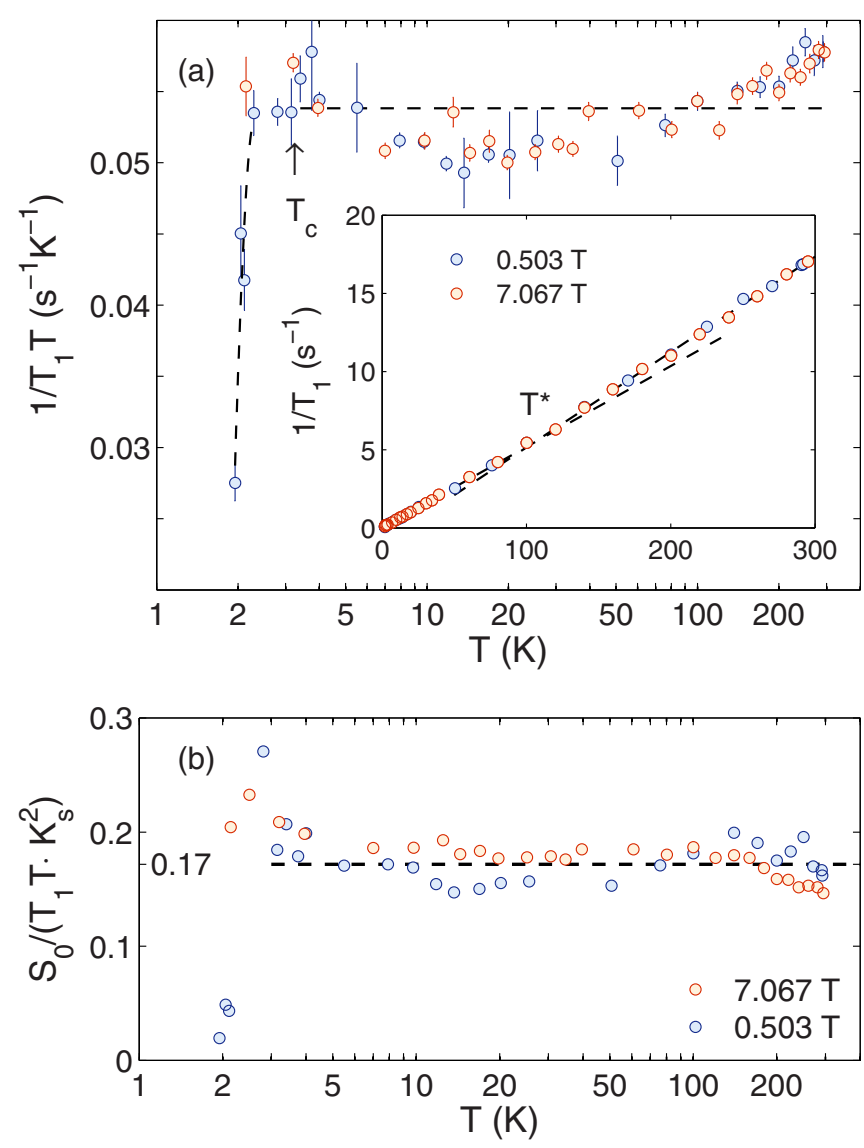

FIG. 12. (a) $1 / T_{1} T$ vs temperature measured at 0.5 and $7 \mathrm{~T}$. Above $T_{c}$ the product is almost constant $\left(0.054 \mathrm{~s}^{-1} \mathrm{~K}^{-1}\right)$. Inset: $1 / T_{1}$ vs temperature measured at the same fields. The field-independent spin-lattice relaxation rate is linear in temperature and changes slope at $T^{\star} \sim 103 \mathrm{~K}$. (b) The Korringa ratio vs temperature is almost constant down to $T_{c}$. An average $\alpha^{-1}$ value much lower than 1 (here shown by a dashed line) suggests ferromagnetic electronic correlations in $\mathrm{Mo}_{3} \mathrm{P}$.

conduction electrons (Mo), a high $\alpha$ value is not unusual (see Ref. [57]) and suggests at most moderate ferromagnetic correlations.

\section{Electronic band structure and discussion}

To further understand the properties of the normal and superconducting states of $\mathrm{Mo}_{3} \mathrm{P}$, we performed DFT bandstructure calculations, whose main results are summarized in Fig. 13. As shown in panels (a)-(c), close to Fermi level the DOS is dominated by the Mo $4 d$ electrons, while the low-lying states near $-6 \mathrm{eV}$ are dominated by contributions from both Mo- $4 d$ and P-3p electrons. The estimated DOS at Fermi level is about 1.56 states/(eV f.u.) $[=12.5$ states $/(\mathrm{eV}$ cell) $/ Z$, with $Z=8$ the number of $\mathrm{Mo}_{3} \mathrm{P}$ formula units per unit cell], which is comparable to the experimental results in Sec. IIIE and Table II. The relatively high DOS hints at a good metallic behavior in $\mathrm{Mo}_{3} \mathrm{P}$, confirmed by both electrical resistivity data and the fast NMR relaxation rates. The electronic band structure calculated without considering the SOC is shown in panel (d), while that including SOC in panel (e). In the former case, there are two almost 


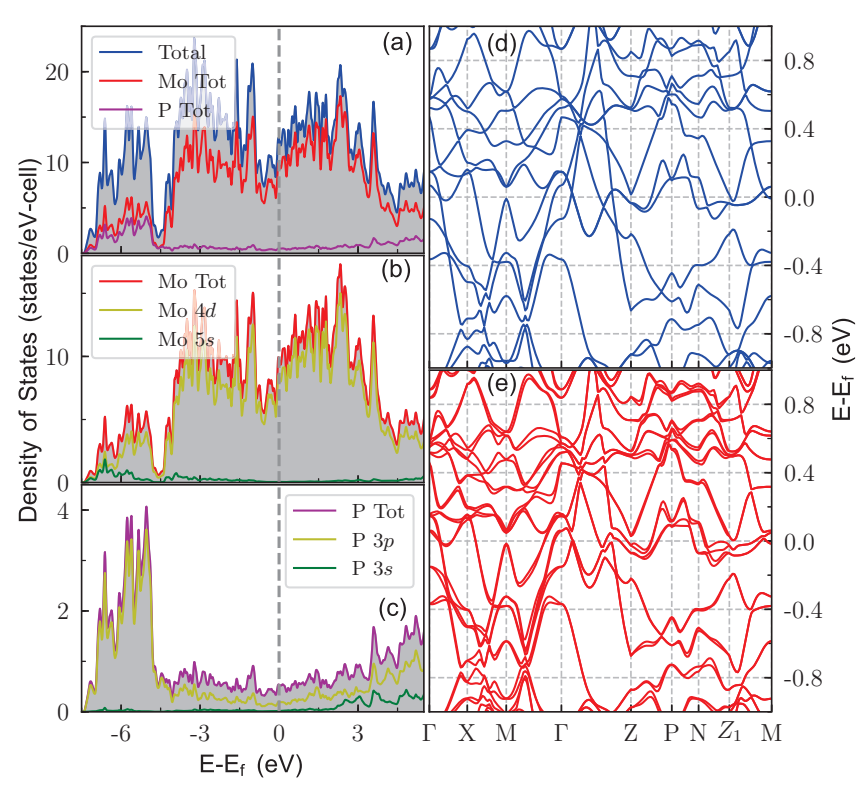

FIG. 13. Calculated electronic band structure for $\mathrm{Mo}_{3} \mathrm{P}$. Total and partial (Mo and P) density of states (a). Orbital-resolved density of states for the Mo (b) and $\mathrm{P}$ atoms (c). Band structure of $\mathrm{Mo}_{3} \mathrm{P}$ without (d) and with SOC interactions (e), calculated within $\pm 1 \mathrm{eV}$ from the Fermi energy level.

overlapping bands at the $Z$ point, about $\sim 0.05 \mathrm{eV}$ below the Fermi level. After including the SOC the bands separate, since SOC breaks the band degeneracy and brings one of the bands closer to the Fermi level [see Fig. 13(e)]. The dispersion of the Mo $4 d$ bands, which cross the Fermi level, is rather small with a bandwidth of about $\sim 0.3-0.4 \mathrm{eV}$. The band splitting due to the ASOC is about $\sim 90 \mathrm{meV}$, a value comparable to that of other NCSCs, e.g., $\mathrm{Y}_{2} \mathrm{C}_{3}$ $(\sim 15 \mathrm{meV})$ [58] and $(\mathrm{Sr}, \mathrm{Ba})(\mathrm{Ni}, \mathrm{Pd}) \mathrm{Si}_{3}(<20 \mathrm{meV})$ [59], but much smaller than that of $\mathrm{CePt}_{3} \mathrm{Si}(\sim 200 \mathrm{meV})$ [60] and $\mathrm{Li}_{2} \mathrm{Pt}_{3} \mathrm{~B}(\sim 200 \mathrm{meV})$ [61]. It is accepted that the band splitting at Fermi level is of primary importance for the superconducting properties [1]. Indeed, because of the large ASOCinduced band splitting, $\mathrm{Li}_{2} \mathrm{Pt}_{3} \mathrm{~B}$ shows nodal superconductivity [62], while the upper critical field in $\mathrm{CePt}_{3} \mathrm{Si}$ exceeds the Pauli limit [63]. Conversely, in $\mathrm{Y}_{2} \mathrm{C}_{3}$ and $(\mathrm{Sr}, \mathrm{Ba})(\mathrm{Ni}, \mathrm{Pd}) \mathrm{Si}_{3}$, the upper critical field is relatively small and they are fully gapped superconductors $[59,64]$. The latter seems also to be the case of $\mathrm{Mo}_{3} \mathrm{P}$, which shows a small band splitting, a low $H_{c 2}$ value, and a singlet-pairing superconducting state.

We also compare the superconductivity of $\mathrm{Mo}_{3} \mathrm{P}$ with other NCSCs. As shown in Fig. 14, the different families of superconductors can be classified according to the ratio of the superconducting transition temperature $T_{c}$ to the effective Fermi temperature $T_{\mathrm{F}}$, in a so-called Uemura plot [66]. Several types of unconventional superconductors, including heavy fermions, organics, high- $T_{c}$ iron pnictides, and cuprates, all lie in a $1 / 100<T_{c} / T_{\mathrm{F}}<1 / 10$ range (gray region). Conversely, conventional BCS superconductors exhibit $T_{c} / T_{\mathrm{F}}<1 / 1000$, here exemplified by the elemental Sn, Al, Zn, and Mo. Most of the NCSCs, e.g., $\mathrm{Re} T, \mathrm{Mo}_{3} \mathrm{Al}_{2} \mathrm{C}, \mathrm{Li}_{2}(\mathrm{Pd}, \mathrm{Pt})_{3} \mathrm{~B}$, and $\mathrm{LaNiC}_{2}$, exhibit a $T_{c} / T_{\mathrm{F}}$ between the unconventional and conventional cases. This is also the case for $\mathrm{Mo}_{3} \mathrm{P}$. According to the super-

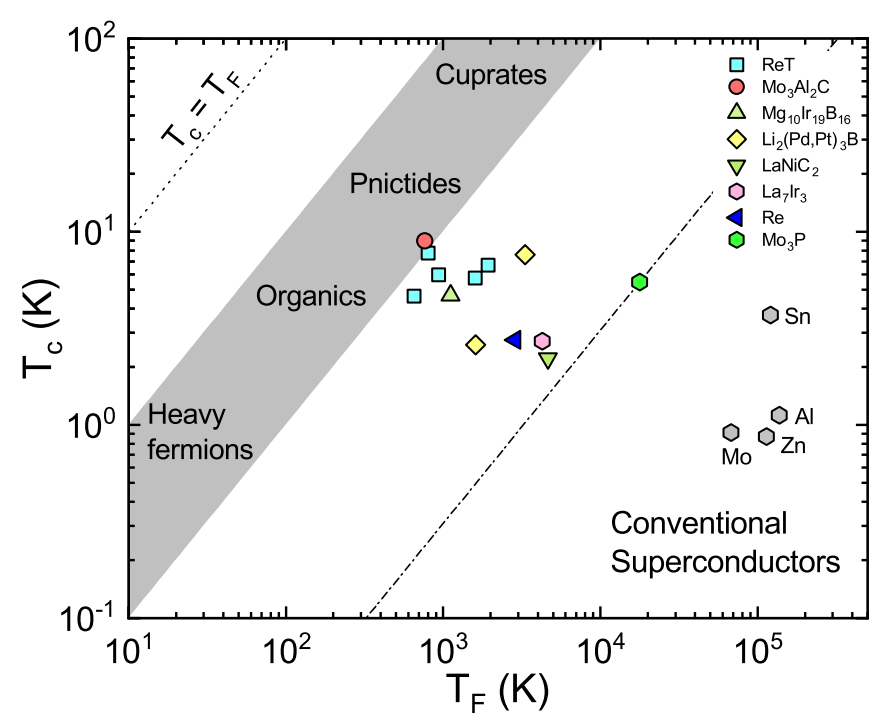

FIG. 14. Uemura plot for different types of superconductors. With $1 / 100<T_{c} / T_{\mathrm{F}}<1 / 10$, the gray region indicates the band of unconventional superconductors, including heavy fermions, organics, fullerenes, pnictides, and high- $T_{c}$ cuprates. Selected noncentrosymmetric and elementary superconductors are shown as symbols (adopted from Refs. $[16,65,66])$. The dotted line corresponds to $T_{c}=$ $T_{\mathrm{F}}$ (here $T_{\mathrm{F}}$ is the temperature associated with the Fermi energy, $E_{\mathrm{F}}=$ $k_{\mathrm{B}} T_{\mathrm{F}}$ ), while the dash-dotted line indicates $T_{c} / T_{\mathrm{F}}=3.09 \times 10^{-4}$ for $\mathrm{Mo}_{3} \mathrm{P}$.

conducting parameters obtained from our measurements (see Table II), we find a $T_{c} / T_{\mathrm{F}}=3.09 \times 10^{-4}$ for $\mathrm{Mo}_{3} \mathrm{P}$, which is significantly enhanced $(\sim 30$ times) compared to elementary Mo $\left(T_{c} / T_{\mathrm{F}}=0.92 / 6.77 \times 10^{4}=1.36 \times 10^{-5}\right)[41]$. On the other hand, $\mathrm{Mo}_{3} \mathrm{P}$ shows a similar $T_{c} / T_{\mathrm{F}}$ value as $\mathrm{LaNiC}_{2}$ and $\mathrm{La}_{7} \mathrm{Ir}_{3}$ (all lying close to the same dash-dotted line). While the latter exhibit unconventional superconductivity with broken TRS [11,12], the TRS is preserved in $\mathrm{Mo}_{3} \mathrm{P}$. Further studies, such as gap-symmetry analysis, are desirable to offer an answer to such different outcomes.

\section{CONCLUSION}

To summarize, we studied the normal- and superconducting-state properties of the $\mathrm{Mo}_{3} \mathrm{P}$ NCSC by means of bulk (electrical resistivity, magnetization, and heat capacity) and local-probe ( $\mu$ SR and NMR) techniques and numerical band-structure calculations. The superconducting state of $\mathrm{Mo}_{3} \mathrm{P}$ is characterized by $T_{c}=5.5 \mathrm{~K}$, a relatively low $\mu_{0} H_{c 2}(0)=0.94 \mathrm{~T}$, and $\kappa \sim 6$. The temperature dependence of the superfluid density and zero-field electronic specific heat reveal a nodeless superconducting state, which is well described by an isotropic s-wave model and is consistent with spin-singlet pairing. The lack of spontaneous magnetic fields below $T_{c}$ indicates that time-reversal symmetry is preserved in the superconducting state of $\mathrm{Mo}_{3} \mathrm{P}$. Electronic band structure calculations suggest a small band splitting due to the ASOC and a sizable density of states at the Fermi level, confirmed also by NMR, which shows $\mathrm{Mo}_{3} \mathrm{P}$ to be a metal with a rather high $N\left(\epsilon_{\mathrm{F}}\right)$ and moderate ferromagnetic electron correlations. 

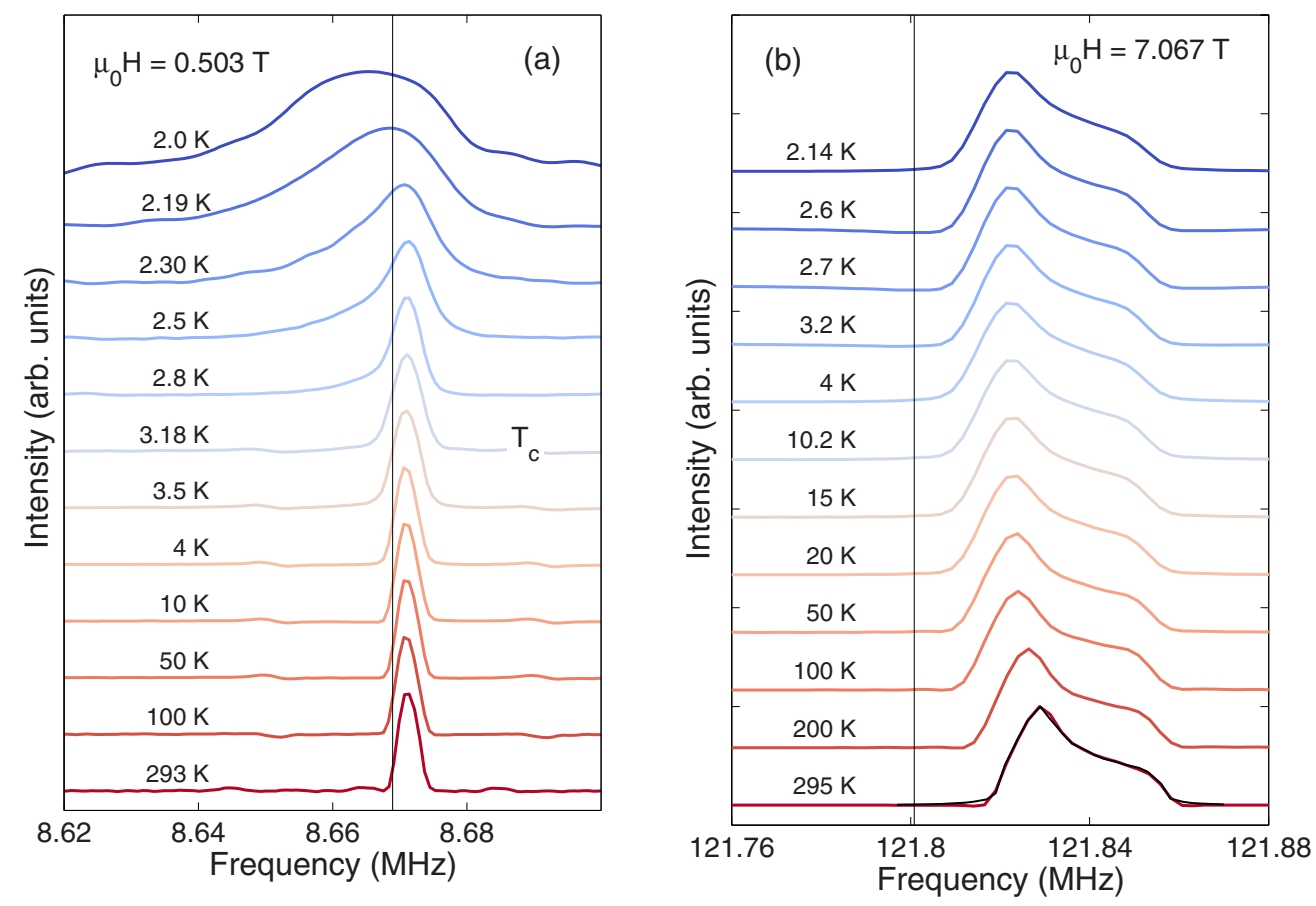

FIG. 15. Selected $\mathrm{Mo}_{3} \mathrm{P}^{31} \mathrm{P}$ NMR line shapes measured at (a) 0.5 and (b) $7 \mathrm{~T}$. While at low field a temperature-dependent line broadening and frequency shift are observable (especially below $T_{c}$ ), at high field the line shapes are practically temperature independent. The vertical lines indicate the ${ }^{31} \mathrm{P}$ NMR reference frequencies at the respective fields. The black line on top of the $295 \mathrm{~K}$ data in panel (b) represents a fit using a Knight-shift anisotropy model (see text for details).

\section{ACKNOWLEDGMENTS}

This work was supported by the Schweizerische Nationalfonds zur Förderung der Wissenschaftlichen Forschung, SNF (Grants No. 200021-169455 and No. 206021-139082). L.J.C. thanks MOST Funding for support under Projects No. 1042112-M-006-010-MY3 and No. 107-2112-M-006-020. We acknowledge assistance from other beamline scientists at the HAL-9500 and GPS $\mu$ SR spectrometers at PSI.

\section{APPENDIX: NMR LINE SHAPES AND RELAXATION RATES}

A selection of ${ }^{31} \mathrm{P}$ NMR line shapes for both magnetic fields $(0.5$ and $7 \mathrm{~T})$ is shown in Fig. 15. At low field (a), the transition to the superconducting phase at low temperature is indicated by a broadening of the line and by its qualitative change of shape. Above $T_{c}$, the line shape is described by a Gaussian (analysis performed by DMFIT), whereas below $T_{c}$, the line shapes can be fitted by means of a convolution of a Gaussian with a Lorentzian. At high field (b), the SC phase transition is suppressed by the applied magnetic field, which also enhances the anisotropy of the line shapes. The black line on top of the $295 \mathrm{~K}$ data represents a fit using a Knightshift anisotropy model (see main text). Here, $\delta_{11}>\delta_{22}>$ $\delta_{33}$ represent the principal components of the shift tensor, identified with the shoulders and the maximum $\left(\delta_{22}\right)$ of the NMR line shapes shown in Fig. 15(b). The fit parameters in the Herzfeld-Berger notation [67] used here are the isotropic component of the shift tensor $\delta_{\text {iso }}$ (i.e., the trace average), the linewidth $\Omega$, and the anisotropy $\eta_{\mathrm{CS}}$ (see main text).
The Clogston-Jaccarino $K-\chi$ plot shown in Fig. 16 clearly indicates that the Knight shift depends linearly on the molar susceptibility $\chi$ in the range $(0.5-1) \times 10^{-4} \mathrm{emu} / \mathrm{mol}$, corresponding to a temperature range $2-50 \mathrm{~K}$. This linear dependency is given by

$$
K(\chi)[\%]=-1.6451 \times 10^{-3} \chi+2.5226 \times 10^{-2} .
$$

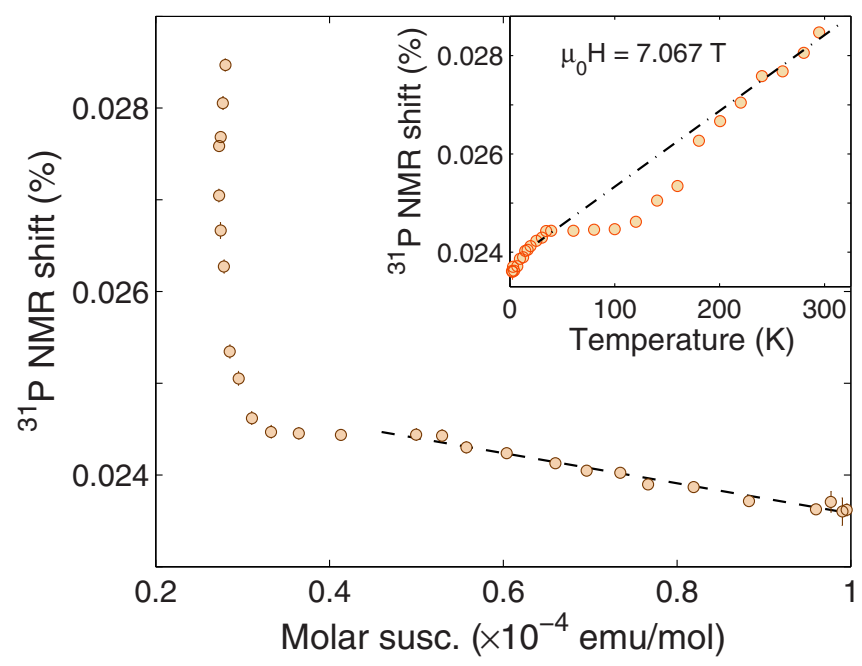

FIG. 16. Clogston-Jaccarino $K-\chi$ plot for $\mathrm{Mo}_{3} \mathrm{P}$ as measured at $7.067 \mathrm{~T}$. The low-temperature part was fitted by using a linear relation, as given in Eq. (A1). Inset: ${ }^{31} \mathrm{P}$ NMR shift vs temperature measured at the same field. The dash-dotted line highlights the shift deviations in the 50-200 K range. 
The offset may indicate the presence of other temperatureindependent components. The coupling constant [i.e., the coefficient in Eq. (A1)] may become temperature dependent for $\chi<0.5 \times 10^{-4} \mathrm{emu} / \mathrm{mol}$ [68]. From the slope $d K / d \chi$ of the $K-\chi$ plot for $T<50 \mathrm{~K}$, by assuming a standard electronic $g$ factor of 2.0, the relation $K=g A_{\mathrm{hf}} \chi+K_{\text {offset }}$ gives a hyperfine coupling constant $A_{\mathrm{hf}}=-0.082 \mathrm{~T} / \mu_{\mathrm{B}}$ and a zero intercept of $0.025 \%$.

As shown in Fig. 16, both the $K-\chi$ plot behavior and the temperature dependence of the NMR shift are rather complicated. This is not unusual for $d$-type electron systems, known for their nonlinear $K(T)$ dependencies (see, e.g., Ref. [69]). In our case, between approximately 30 and $200 \mathrm{~K}$, the Knight shift deviates markedly from linearity to exhibit a shallow minimum around $100 \mathrm{~K}$. At $30 \mathrm{~K}, 1 /\left(T_{1} T\right)$ exhibits a minimum [see Fig. 12(a)], while around $100 \mathrm{~K}, 1 / T_{1}$ changes slope. Since these anomalies appear in rather independent types of measurements $\left(K\right.$ and $\left.T_{1}\right)$, they might represent true modifications of the $\mathrm{Mo}_{3} \mathrm{P}$ electronic properties. Although currently an explanation concerning their nature is missing, future studies of the Hall coefficient vs temperature might help to better determine their origin.
[1] E. Bauer and M. Sigrist, editors, Non-Centrosymmetric Superconductors, Lecture Notes in Physics (Springer, Berlin, 2012), Vol. 847.

[2] M. Smidman, M. B. Salamon, H. Q. Yuan, and D. F. Agterberg, Superconductivity and spin-orbit coupling in noncentrosymmetric materials: A review, Rep. Prog. Phys. 80, 036501 (2017).

[3] K. Ishida, H. Mukuda, Y. Kitaoka, K. Asayama, Z. Q. Mao, Y. Mori, and Y. Maeno, Spin-triplet superconductivity in $\mathrm{Sr}_{2} \mathrm{RuO}_{4}$ identified by ${ }^{17} \mathrm{O}$ Knight shift, Nature (London) 396, 658 (1998).

[4] H. Tou, Y. Kitaoka, K. Ishida, K. Asayama, N. Kimura, Y. Ōnuki, E. Yamamoto, Y. Haga, and K. Maezawa, Nonunitary Spin-Triplet Superconductivity in $\mathrm{UPt}_{3}$ : Evidence from ${ }^{195} \mathrm{Pt}$ Knight Shift Study, Phys. Rev. Lett. 80, 3129 (1998).

[5] A. Mackenzie and Y. Maeno, The superconductivity of $\mathrm{Sr}_{2} \mathrm{RuO}_{4}$ and the physics of spin-triplet pairing, Rev. Mod. Phys. 75, 657 (2003), and references therein.

[6] R. Joynt and L. Taillefer, The superconducting phases of $\mathrm{UPt}_{3}$, Rev. Mod. Phys. 74, 235 (2002), and references therein.

[7] G. M. Luke, A. Keren, L. P. Le, W. D. Wu, Y. J. Uemura, D. A. Bonn, L. Taillefer, and J. D. Garrett, Muon Spin Relaxation in $\mathrm{UPt}_{3}$, Phys. Rev. Lett. 71, 1466 (1993).

[8] G. M. Luke, Y. Fudamoto, K. M. Kojima, M. I. Larkin, J. Merrin, B. Nachumi, Y. J. Uemura, Y. Maeno, Z. Q. Mao, Y. Mori, H. Nakamura, and M. Sigrist, Time-reversal symmetrybreaking superconductivity in $\mathrm{Sr}_{2} \mathrm{RuO}_{4}$, Nature (London) 394, 558 (1998).

[9] J. Xia, Y. Maeno, P. T. Beyersdorf, M. M. Fejer, and A. Kapitulnik, High Resolution Polar Kerr Effect Measurements of $\mathrm{Sr}_{2} \mathrm{RuO}_{4}$ : Evidence for Broken Time-Reversal Symmetry in the Superconducting State, Phys. Rev. Lett. 97, 167002 (2006).

[10] E. R. Schemm, W. J. Gannon, C. M. Wishne, W. P. Halperin, and A. Kapitulnik, Observation of broken time-reversal symmetry in the heavy-fermion superconductor $\mathrm{UPt}_{3}$, Science 345, 190 (2014).

[11] A. D. Hillier, J. Quintanilla, and R. Cywinski, Evidence for Time-Reversal Symmetry Breaking in the Noncentrosymmetric Superconductor $\mathrm{LaNiC}_{2}$, Phys. Rev. Lett. 102, 117007 (2009).

[12] J. A. T. Barker, D. Singh, A. Thamizhavel, A. D. Hillier, M. R. Lees, G. Balakrishnan, D. M. Paul, and R. P. Singh, Unconventional Superconductivity in $\mathrm{La}_{7} \mathrm{Ir}_{3}$ Revealed by Muon Spin Relaxation: Introducing a New Family of Noncentrosymmetric Superconductor that Breaks Time-Reversal Symmetry, Phys. Rev. Lett. 115, 267001 (2015).
[13] R. P. Singh, A. D. Hillier, B. Mazidian, J. Quintanilla, J. F. Annett, D. M. Paul, G. Balakrishnan, and M. R. Lees, Detection of Time-Reversal Symmetry Breaking in the Noncentrosymmetric Superconductor $\operatorname{Re}_{6} \mathrm{Zr}$ Using Muon-Spin Spectroscopy, Phys. Rev. Lett. 112, 107002 (2014).

[14] D. Singh, J. A. T. Barker, A. Thamizhavel, D. M. Paul, A. D. Hillier, and R. P. Singh, Time-reversal symmetry breaking in the noncentrosymmetric superconductor $\mathrm{Re}_{6} \mathrm{Hf}$ : Further evidence for unconventional behavior in the $\alpha$-Mn family of materials, Phys. Rev. B 96, 180501(R) (2017).

[15] T. Shang, G. M. Pang, C. Baines, W. B. Jiang, W. Xie, A. Wang, M. Medarde, E. Pomjakushina, M. Shi, J. Mesot, H. Q. Yuan, and T. Shiroka, Nodeless superconductivity and time-reversal symmetry breaking in the noncentrosymmetric superconductor $\mathrm{Re}_{24}$ Ti, Phys. Rev. B 97, 020502(R) (2018).

[16] T. Shang, M. Smidman, S. K. Ghosh, C. Baines, L. J. Chang, D. J. Gawryluk, J. A. T. Barker, R. P. Singh, D. M. Paul, G. Balakrishnan, E. Pomjakushina, M. Shi, M. Medarde, A. D. Hillier, H. Q. Yuan, J. Quintanilla, J. Mesot, and T. Shiroka, Time-Reversal Symmetry Breaking in Re-Based Superconductors, Phys. Rev. Lett. 121, 257002 (2018).

[17] J. Quintanilla, A. D. Hillier, J. F. Annett, and R. Cywinski, Relativistic analysis of the pairing symmetry of the noncentrosymmetric superconductor $\mathrm{LaNiC}_{2}$, Phys. Rev. B 82, 174511 (2010).

[18] A. D. Hillier, J. Quintanilla, B. Mazidian, J. F. Annett, and R. Cywinski, Nonunitary Triplet Pairing in the Centrosymmetric Superconductor LaNiGa 2 , Phys. Rev. Lett. 109, 097001 (2012).

[19] Z. F. Weng, J. L. Zhang, M. Smidman, T. Shang, J. Quintanilla, J. F. Annett, M. Nicklas, G. M. Pang, L. Jiao, W. B. Jiang, Y. Chen, F. Steglich, and H. Q. Yuan, Two-Gap Superconductivity in $\mathrm{LaNiGa}_{2}$ with Nonunitary Triplet Pairing and Even Parity Gap Symmetry, Phys. Rev. Lett. 117, 027001 (2016).

[20] S. Ghosh, J. F. Annett, and J. Quintanilla, Time-reversal symmetry breaking in superconductors through loop Josephsoncurrent order, arXiv:1803.02618.

[21] B. T. Matthias, E. Corenzwit, and C. E. Miller, Superconducting compounds, Phys. Rev. 93, 1415 (1954).

[22] R. D. Blaugher, J. K. Hulm, and P. N. Yocom, Superconducting phosphides of the transition metals, J. Phys. Chem. Solids 26, 2037 (1965).

[23] B. Sellberg and S. Rundqvist, The crystal structure of $\mathrm{Mo}_{3} \mathrm{P}$, Acta Chem. Scand. 19, 760 (1965). 
[24] A. Amato, H. Luetkens, K. Sedlak, A. Stoykov, R. Scheuermann, M. Elender, A. Raselli, and D. Graf, The new versatile general purpose surface-muon instrument (GPS) based on silicon photomultipliers for $\mu \mathrm{SR}$ measurements on a continuous-wave beam, Rev. Sci. Instrum. 88, 093301 (2017).

[25] A. A. Suter and B. M. Wojek, Musrfit: A free platformindependent framework for $\mu$ SR data analysis, Phys. Procedia 30, 69 (2012).

[26] P. Blaha, K. Schwarz, G. K. H. Madsen, D. Kvasnicka, and J. Luitz, WIEN2k, An Augmented Plane Wave + Local Orbitals Program for Calculating Crystal Properties (Karlheinz Schwarz, Techn. Universität Wien, Austria, 2001).

[27] J. P. Perdew, K. Burke, and M. Ernzerhof, Generalized Gradient Approximation Made Simple, Phys. Rev. Lett. 77, 3865 (1996).

[28] J. Rodríguez-Carvajal, Recent advances in magnetic structure determination by neutron powder diffraction, Physica B (Amsterdam) 192, 55 (1993).

[29] F. Bloch, Zum elektrischen Widerstandsgesetz bei tiefen Temperaturen, Z. Phys. 59, 208 (1930).

[30] F. J. Blatt, Physics of Electronic Conduction in Solids (McGrawHill, New York, 1968), pp. 185-190.

[31] N. F. Mott and H. Jones, The Theory of the Properties of Metals and Alloys (Oxford University Press, London, 1958).

[32] N. F. Mott, Electrons in transition metals, Adv. Phys. 13, 325 (1964).

[33] X. Zhu, H. Yang, L. Fang, G. Mu, and H.-H. Wen, Upper critical field, Hall effect and magnetoresistance in the ironbased layered superconductor $\mathrm{LaFeAsO}_{0.9} \mathrm{~F}_{0.1-\delta}$, Supercond. Sci. Technol. 21, 105001 (2008).

[34] N. R. Werthamer, E. Helfand, and P. C. Hohenberg, Temperature and purity dependence of the superconducting critical field, $H_{c 2}$. III. Electron spin and spin-orbit effects, Phys. Rev. 147, 295 (1966).

[35] F. Kneidinger, H. Michor, A. Sidorenko, E. Bauer, I. Zeiringer, P. Rogl, C. Blaas-Schenner, D. Reith, and R. Podloucky, Synthesis, characterization, electronic structure, and phonon properties of the noncentrosymmetric superconductor LaPtSi, Phys. Rev. B 88, 104508 (2013).

[36] D. C. Peets, A. Maldonado, M. Enayat, Z. Sun, P. Wahl, and A. P. Schnyder, Upper critical field of the noncentrosymmetric superconductor BiPd, Phys. Rev. B 93, 174504 (2016).

[37] N. Kimura, N. Kabeya, K. Saitoh, K. Satoh, H. Ogi, K. Ohsaki, and H. Aoki, Type II/1 superconductivity with extremely high $H_{c 3}$ in noncentrosymmetric $\mathrm{LaRhSi}_{3}$, J. Phys. Soc. Jpn. 85, 024715 (2016).

[38] V. K. Anand, D. Britz, A. Bhattacharyya, D. T. Adroja, A. D. Hillier, A. M. Strydom, W. Kockelmann, B. D. Rainford, and K. A. McEwen, Physical properties of noncentrosymmetric superconductor $\mathrm{LaIrSi}_{3}$ : A $\mu$ SR study, Phys. Rev. B 90, 014513 (2014).

[39] M. Smidman, A. D. Hillier, D. T. Adroja, M. R. Lees, V. K. Anand, R. P. Singh, R. I. Smith, D. M. Paul, and G. Balakrishnan, Investigations of the superconducting states of noncentrosymmetric $\mathrm{LaPdSi}_{3}$ and $\mathrm{LaPtSi}_{3}$, Phys. Rev. B 89, 094509 (2014).

[40] E. H. Brandt, Properties of the ideal Ginzburg-Landau vortex lattice, Phys. Rev. B 68, 054506 (2003).

[41] C. Kittel, Introduction to Solid State Physics, 8th ed. (John Wiley \& Sons, Hoboken, NJ, 2005).
[42] W. L. McMillan, Transition temperature of strong-coupled superconductors, Phys. Rev. 167, 331 (1968).

[43] R. I. Boughton, J. L. Olsen, and C. Palmy, Pressure effects in superconductors, in Prog. Low Temp. Phys. 6, 163 (1970).

[44] T. Shang, D. J. Gawryluk, J. A. T. Verezhak, E. Pomjakushina, M. Shi, M. Medarde, J. Mesot, and T. Shiroka, Structure and superconductivity in the binary $\mathrm{Re}_{1-x} \mathrm{Mo}_{x}$ alloys, Phys. Rev. Mater. 3, 024801 (2019).

[45] M. Tinkham, Introduction to Superconductivity, 2nd ed. (Dover Publications, Mineola, NY, 1996).

[46] H. Padamsee, J. E. Neighbor, and C. A. Shiffman, Quasiparticle phenomenology for thermodynamics of strong-coupling superconductor, J. Low Temp. Phys. 12, 387 (1973).

[47] A. Maisuradze, R. Khasanov, A. Shengelaya, and H. Keller, Comparison of different methods for analyzing $\mu$ SR line shapes in the vortex state of type-II superconductors, J. Phys.: Condens. Matter 21, 075701 (2009), and references therein.

[48] W. Barford and J. M. F. Gunn, The theory of the measurement of the London penetration depth in uniaxial type II superconductors by muon spin rotation, Phys. C (Amsterdam) 156, 515 (1988).

[49] A. Carrington and F. Manzano, Magnetic penetration depth of $\mathrm{MgB}_{2}$, Physica C (Amsterdam) 385, 205 (2003).

[50] Y. Aoki, A. Tsuchiya, T. Kanayama, S. R. Saha, H. Sugawara, H. Sato, W. Higemoto, A. Koda, K. Ohishi, K. Nishiyama, and R. Kadono, Time-Reversal Symmetry-Breaking Superconductivity in Heavy-Fermion $\mathrm{PrOs}_{4} \mathrm{Sb}_{12}$ detected by muon-spin relaxation, Phys. Rev. Lett. 91, 067003 (2003).

[51] R. Kubo and T. Toyabe, A stochastic model for low field resonance and relaxation, in Magnetic Resonance and Relaxation, edited by R. Blinc (North-Holland, Amsterdam, 1967), pp. 810-823.

[52] A. Yaouanc and P. D. de Réotier, Muon Spin Rotation, Relaxation, and Resonance: Applications to Condensed Matter (Oxford University Press, Oxford, 2011).

[53] D. Massiot, F. Fayon, M. Capron, I. King, S. Le Calvé, B. Alonso, J.-O. Durand, B. Bujoli, Z. Gan, and G. Hoatson, Modelling one- and two-dimensional solid-state NMR spectra, Magn. Reson. Chem. 40, 70 (2002).

[54] N. Barbero, T. Shiroka, B. Delley, T. Grant, A. J. S. Machado, Z. Fisk, H.-R. Ott, and J. Mesot, Doping-induced superconductivity of $\mathrm{ZrB}_{2}$ and $\mathrm{HfB}_{2}$, Phys. Rev. B 95, 094505 (2017).

[55] J. Korringa, Nuclear magnetic relaxation and resonance line shift in metals, Physica (Amsterdam) 16, 601 (1950).

[56] Y. Yafet and V. Jaccarino, Nuclear spin relaxation in transition metals; core polarization, Phys. Rev. 133, A1630 (1964).

[57] A. Narath and H. T. Weaver, Effects of electron-electron interactions on nuclear spin-lattice relaxation rates and Knight shifts in alkali and noble metals, Phys. Rev. 175, 373 (1968).

[58] Y. Nishikayama, T. Shishidou, and T. Oguchi, Electronic properties of $\mathrm{Y}_{2} \mathrm{C}_{3}$ by first-principles calculations, J. Phys. Soc. Jpn. 76, 064714 (2007).

[59] F. Kneidinger, L. Salamakha, E. Bauer, I. Zeiringer, P. Rogl, C. Blaas-Schenner, D. Reith, and R. Podloucky, Superconductivity in noncentrosymmetric $\mathrm{BaAl}_{4}$ derived structures, Phys. Rev. B 90, 024504 (2014).

[60] K. V. Samokhin, E. S. Zijlstra, and S. K. Bose, $\mathrm{CePt}_{3} \mathrm{Si}$ : An unconventional superconductor without inversion center, Phys. Rev. B 69, 094514 (2004). 
[61] K.-W. Lee and W. E. Pickett, Crystal symmetry, electronphonon coupling, and superconducting tendencies in $\mathrm{Li}_{2} \mathrm{Pd}_{3} \mathrm{~B}$ and $\mathrm{Li}_{2} \mathrm{Pt}_{3} \mathrm{~B}$, Phys. Rev. B 72, 174505 (2005).

[62] H. Q. Yuan, D. F. Agterberg, N. Hayashi, P. Badica, D. Vandervelde, K. Togano, M. Sigrist, and M. B. Salamon, $S$ Wave Spin-Triplet Order in Superconductors without Inversion Symmetry: $\mathrm{Li}_{2} \mathrm{Pd}_{3} \mathrm{~B}$ and $\mathrm{Li}_{2} \mathrm{Pt}_{3} \mathrm{~B}$, Phys. Rev. Lett. 97, 017006 (2006).

[63] E. Bauer, G. Hilscher, H. Michor, C. Paul, E. W. Scheidt, A. Gribanov, Y. Seropegin, H. Noël, M. Sigrist, and P. Rogl, Heavy Fermion Superconductivity and Magnetic Order in Noncentrosymmetric $\mathrm{CePt}_{3} \mathrm{Si}$, Phys. Rev. Lett. 92, 027003 (2004).

[64] S. Kuroiwa, Y. Saura, J. Akimitsu, M. Hiraishi, M. Miyazaki, K. H. Satoh, S. Takeshita, and R. Kadono, Multigap Superconductivity in Sesquicarbides $\mathrm{La}_{2} \mathrm{C}_{3}$ and $\mathrm{Y}_{2} \mathrm{C}_{3}$, Phys. Rev. Lett. 100, 097002 (2008).

[65] J. A. T. Barker, B. D. Breen, R. Hanson, A. D. Hillier, M. R. Lees, G. Balakrishnan, D. M. Paul, and R. P. Singh, Superconducting and normal-state properties of the noncentrosymmetric superconductor $\mathrm{Re}_{3}$ Ta, Phys. Rev. B 98, 104506 (2018), and references therein.

[66] Y. J. Uemura, L. P. Le, G. M. Luke, B. J. Sternlieb, W. D. Wu, J. H. Brewer, T. M. Riseman, C. L. Seaman, M. B. Maple, M. Ishikawa, D. G. Hinks, J. D. Jorgensen, G. Saito, and H. Yamochi, Basic Similarities among Cuprate, Bismuthate, Organic, Chevrel-Phase, and Heavy-Fermion Superconductors Shown by Penetration-Depth Measurements, Phys. Rev. Lett. 66, 2665 (1991).

[67] J. Herzfeld and A. E. Berger, Sideband intensities in NMR spectra of samples spinning at the magic angle, J. Chem. Phys. 73, 6021 (1980).

[68] P.-C. Ho, D. E. MacLaughlin, L. Shu, O. O. Bernal, S. Zhao, A. A. Dooraghi, T. Yanagisawa, M. B. Maple, and R. H. Fukuda, Muon spin rotation and relaxation in $\mathrm{Pr}_{1-x} \mathrm{Nd}_{x} \mathrm{Os}_{4} \mathrm{Sb}_{12}$ : Paramagnetic states, Phys. Rev. B 89, 235111 (2014).

[69] G. S. Carter, L. H. Bennett, and D. J. Kahan, Metallic Shifts in NMR (Pergamon Press, Oxford, 1977). 